\title{
DIGITALCOMMONS
}

$@$ WAYNESTATE-

Wayne State University

\section{Optimization and Equilibrium Problems with Equilibrium Constraints in Infinite-Dimensional Spaces}

Boris S. Mordukhovich

Wayne State University, boris@math.wayne.edu

\section{Recommended Citation}

Mordukhovich, Boris S., "Optimization and Equilibrium Problems with Equilibrium Constraints in Infinite-Dimensional Spaces" (2006). Mathematics Research Reports. Paper 33.

http://digitalcommons.wayne.edu/math_reports/33

This Technical Report is brought to you for free and open access by the Mathematics at DigitalCommons@WayneState. It has been accepted for inclusion in Mathematics Research Reports by an authorized administrator of DigitalCommons@WayneState. 


\section{OPTIMIZATION AND EQUILIBRIUM PROBLEMS WITH EQUILIBRIUM CONSTRAINTS IN INFINITE- DIMENSIONAL SPACES}

BORIS S. MORDUKHOVICH

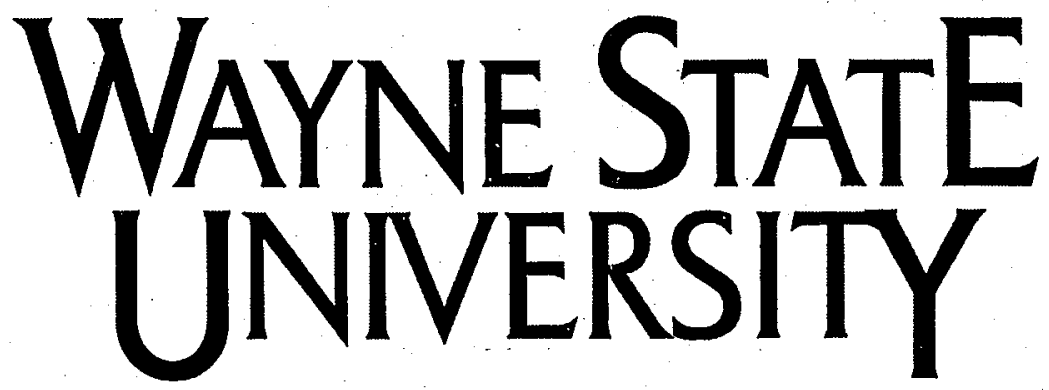

Detroit, MI 48202

Department of Mathematics

Research Report

2006 Series

\#3 


\title{
OPTIMIZATION AND EQUILIBRIUM PROBLEMS WITH EQUILIBRIUM CONSTRAINTS IN INFINITE-DIMENSIONAL SPACES ${ }^{1}$
}

\author{
B. S. MORDUKHOVICH ${ }^{2}$
}

\section{Dedicated to Steve Robinson in honor of his 65th birthday}

\begin{abstract}
The paper is devoted to applications of modern variational analysis to the study of constrained optimization and equilibrium problems in infinite-dimensional spaces. We pay a particular attention to the remarkable classes of optimization and equilibrium problems identified as MPECs (mathematical programs with equilibrium constraints) and EPECs (equilibrium problems with equilibrium constraints) treated from the viewpoint of multiobjective optimization. Their underlying feature is that the major constraints are governed by parametric generalized equations/variational conditions in the sense of Robinson. Such problems are intrinsically nonsmooth and can be handled by using an appropriate machinery of generalized differentiation exhibiting a rich/full calculus. The case of infinite-dimensional spaces is significantly more involved in comparison with finite dimensions, requiring in addition a certain sufficient amount of compactness and an efficient calculus of the corresponding "sequential normal compactness" (SNC) properties.
\end{abstract}

Key words. nonsmooth and multiobjective optimization-equilibrium constraints-variational analysisgeneralized differentiation-necessary optimality conditions-exact penalization-infinite dimensions

Mathematics Subject Classification (2000): 90C29, 90C30, 49J52, 49J53, 49K27

\section{Introduction}

The main objective of this paper is to study constrained optimization (including vector/multiobjective optimization) problems, which have constraints of the type

$$
0 \in q(x, y)+Q(x, y)
$$

among possible constraints of other kinds. In (1.1), $q: X \times Y \rightarrow P$ is a single-valued mapping while $Q: X \times Y \Rightarrow P$ is a set-valued mapping between Banach spaces, $y \in Y$ stands for the decision variable, and $x \in X$ is a parameter. Models of type (1.1) were introduced by Robinson [26] in the end of 1970s, and since that time they have played a crucial role in many aspects of optimization and variational analysis. It seems that the original motivation for Robinson was to describe variational inequalities and complementarity problems in the form of "generalized equations,". which are distinguished from standard equations by the presence of the multivalued term $Q$ while allowing one to explore this similarity for their qualitative study and numerical solution. Indeed, generalized equations (1.1) reduce to the parametric variational inequalities

$$
\text { find } y \in \Omega \text { with }\langle q(x, y), v-y\rangle \geq 0 \text { for all } v \in \Omega
$$

\footnotetext{
${ }^{1}$ Research was partially supported by the National Science Foundation under grant DMS-0304989 and by the Australian Research Council under grant DP-0451168.

${ }^{2}$ Department of Mathematics, Wayne State University, Detroit, Michigan 48202, USA; boris@math.wayne.edu
} 
when $Q(y)=N(y ; \Omega)$ in $(1.1)$ is the classical normal-cone mapping to a convex set $\Omega$. Based on formalism (1.1), Robinson and his followers developed strong results in sensitivity analysis and numerical methods of solving variational inequalities, complementarity and optimization problems, etc.; see particularly the seminal papers by Robinson [26, 27], his recent survey [29], and the fundamental 2-volume monograph by Facchinei and Pang [6].

It has been well realized that constraints (1.1) can describe certain equilibrium conditions, in particular, those arising from the solution of lower-level parametric problems in hierarchical optimization (e.g., in bilevel programming). On this basis, minimization problems subject to constraints of type (1.1), which express sets of feasible solutions to the upper level of hierarchical optimization; are called mathematical programs with equilibrium constraints (MPECs); see the books by Luo, Pang, and Ralph [13] and by Outrata, Kočvara and Zowe [24] for various approaches and results for such problems; more recent extensive bibliographies and commentaries on MPECs can be found in $[3,6,17]$.

Quite recently, a new class of problems has drawn attention of both researchers and practitioners. This class is generally related to seeking equilibria subject to equilibrium constraints, i.e., to considering problems with equilibrium conditions appearing in both costs and constraints (in other words, on both lower and upper level of hierarchy). Such problems are known as equilibrium problems with equilibrium constraints (EPECs); this term was coined by Stefan Scholtes in his talk at the 3rd International Conference on Complementarity Problems (2002, Cambridge, UK). We refer the reader to the papers by Fukushima and Pang [7], Hu and Ralph [9], and Outrata [23] for more discussions and other references on this class of problems with results mostly related to Nash equilibrium on the upper (or both lower and upper) level.

Another approach to the study of EPECs was suggested by the author [15] from the viewpoint of multiobjective optimization on the upper level of hierarchy, with general equilibrium constraints of type (1.1) on the lower level. This approach is more suitable for deriving optimality conditions in EPECs with Pareto-type equilibria (and other concepts of "generalized order optimality" and "closed preference relations" as given below) on the upper level; cf. also Ye and Zhu [32] for necessary conditions in certain multiobjective problems with variational inequality constraints, where the upper-level optimality is defined by some "regular" preference relations. The recent paper by Mordukhovich, Outrata and Červinka [19] contains efficient implementations and developments of the approach in [15] to an important class of EPECs governed by complementarity conditions on the lower level and the classical weak Pareto optimality on the upper level of hierarchy, with applications to the oligopolistic market modeling. We refer the reader to the book [17] for more discussions on EPECs and related multiobjective problems with various constraints.

Observe that all the results obtained in $[15,19,32]$, as well as those in $[7,9,23]$, concern EPECs in finite-dimensional spaces. The problems considered in [15, 19, 23, 32], being intrinsically nonsmooth, were treated via the generalized differential constructions of variational analysis and calculus rules in finite-dimensional spaces developed earlier by the author. The infinite-dimensional settings considered in this paper are significantly different from their finite-dimensional counterparts from both conceptional and technical viewpoints; see $[16,17]$. One of the principal new ingredients of the infinite-dimensional theory is the 
necessity to deal with "lack of compactness" in infinite dimensions, which requires the usage of certain appropriate "normal compactness" properties and workable rules of their calculus.

The primary goal of this paper is to derive efficient necessary optimality conditions for general infinite-dimensional EPECs and MPECs on the base of the advanced generalized differentiation theory of variational analysis in infinite-dimensional spaces.

The rest of the paper is organized as follows. Section 2 presents a brief review of the basic generalized differential constructions of variational analysis and normal compactness properties needed for formulations and proofs of the main results.

Section 3 is devoted to the study of infinite-dimensional EPECs from the viewpoint of multiobjective optimization with equilibrium constraints of type (1.1) on the lower level and equilibrium relations on the upper level given by "generalized order optimality." The results obtained are based on the extremal principle of variational analysis, which plays a fundamental role in the nonconvex variational theory and applications similarly to convex separation theorems under convexity assumptions. Its infinite-dimensional version in product spaces happens to be the most appropriate for applications to EPECs.

In Section 4 we study some classes of EPECs in infinite-dimensional spaces with equilibrium criteria on the upper level given by "closed preference relations." This eventually requires different tools of generalized differentiation and versions of the extremal principle in comparison with problems from Section 3, while leading to a series of independent results in problems of multiobjective optimization and EPECs. Certain special structures of equilibrium constraints are studied in more detail.

In the final Section 5 we consider a general class of infinite-dimensional MPECs and develop an approach to deriving necessary optimality conditions based on "exact penalization" procedure combining with appropriate tools of generalized differentiation. In finite dimensions, this approach goes back to Ye and Ye [31] and Outrata [22], while the infinitedimensional case under consideration happens to be significantly more involved and offers a larger variety of qualification and optimality conditions. Note that the notion of "calmness" (or "upper-Lipschitzian" property), which is essentially due to Robinson [26, 28], plays a crucial role in this approach.

Our notation is basically standard; see $[16,17]$. Recall that, given a set-valued mapping $F: X \Rightarrow X^{*}$ between a Banach space $X$ and its topological dual $X^{*}$, the sequential PainlevéKuratowski upper/outer limit of $F$ as $x \rightarrow \bar{x}$ with respect to the norm topology of $X$ and the weak ${ }^{*}$ topology $w^{*}$ of $X^{*}$ is

$$
\begin{aligned}
& \underset{x \rightarrow \bar{x}}{\operatorname{Limsup}} F(x):=\left\{x^{*} \in X^{*} \mid \exists \text { sequences } x_{k} \rightarrow \bar{x} \text { and } x_{k}^{*} \stackrel{w^{*}}{\rightarrow} x^{*}\right. \\
& \text { with } \left.x_{k}^{*} \in F\left(x_{k}\right) \text { for all } k \in \mathbb{N}\right\} \text {, }
\end{aligned}
$$

where $\mathbb{N}:=\{1,2, \ldots\}$. Recall also that the symbols $x \stackrel{\Omega}{\rightarrow} \bar{x}$ and $x \stackrel{\varphi}{\rightarrow} \bar{x}$ signify, respectively, that $x \rightarrow \bar{x}$ with $x \in \Omega$ and that $x \rightarrow \bar{x}$ with $\varphi(x) \rightarrow \varphi(\bar{x})$ for sets $\Omega \subset X$ and extendedreal-valued functions $\varphi: X \rightarrow \overline{\mathbb{R}}:=[-\infty, \infty]$. Unless otherwise stated, all the spaces under consideration are Banach with the norm $\|\cdot\|$ and the canonical pairing $\langle\cdot, \cdot\rangle$ between the space in question and its dual. We use $\mathbb{B}_{X}$ to denote the closed unit ball of $X$, where the subindex " $X$ " is omitted when there is no confusion; $\mathbb{B}^{*}$ stands for the closed unit ball of the dual space in question. 


\section{Preliminaries in Variational Analysis}

We start with a brief review of the basic generalized differential constructions of variational analysis and some of their properties widely used in what follows. This is taken from the author's book [16], where the reader can find a comprehensive theory for these constructions with extensive discussions, references, and commentaries.

Developing a geometric approach to generalized differentiation, let us first define the (basic, limiting) normal cone to $\Omega \subset X$ at $\bar{x} \in \Omega$ by

$$
N(\bar{x} ; \Omega):=\underset{\substack{x \rightarrow \bar{x} \\ \varepsilon \downarrow 0}}{\operatorname{Limsup}} \widehat{N}_{\varepsilon}(x ; \Omega)
$$

where $\widehat{N}_{\varepsilon}(x ; \Omega)$ stands for the set of $\varepsilon$-normals $(\varepsilon \geq 0)$ to $\Omega$ at $x \in X$ given by

$$
\widehat{N}_{\varepsilon}(x ; \Omega):=\left\{x^{*} \in X^{*} \mid \underset{\substack{\Omega \\ \limsup _{x}}}{\lim \left(x^{*}, u-x\right\rangle} \leq \varepsilon\right\}, \quad x \in \Omega
$$

and $\widehat{N}_{\varepsilon}(x ; \Omega):=\emptyset$ if $x \notin \Omega$. If the space $X$ is $A$ splund (i.e., each of its separable subspace has a separable dual) and if the set $\Omega$ is locally closed around $\bar{x}$, then we can equivalently put $\varepsilon=0$ in (2.1) and replace $\widehat{N}_{\varepsilon}$ by the generally smaller prenormal (or Fréchet normal) cone $\widehat{N}(x ; \Omega):=\widehat{N}_{0}(x ; \Omega)$. Observe that the class of Asplund spaces is sufficiently large particularly including every reflexive Banach space and every space with a separable dual; see, e.g., the book by Phelps [25] for more details and references.

Given a set-valued mapping $F: X \Rightarrow Y$ and a point $(\bar{x}, \bar{y})$ from its graph

$$
\operatorname{gph} F:=\{(x, y) \in X \times Y \mid y \in F(x)\}
$$

consider two kinds of limiting coderivatives of $F$ at $(\bar{x}, \bar{y})$ : the normal coderivative

$$
D_{N}^{*} F(\bar{x}, \bar{y})\left(\bar{y}^{*}\right):=\left\{x^{*} \in X^{*} \mid\left(x^{*},-y^{*}\right) \in N((\bar{x}, \bar{y}) ; \operatorname{gph} F)\right\}, \quad \bar{y}^{*} \in Y^{*},
$$

and the mixed coderivative

$$
D_{M}^{*} F(\tilde{x}, \bar{y})\left(\bar{y}^{*}\right):=\operatorname{Limsup}_{\substack{(x, y) \rightarrow(\tilde{x}, \bar{y}) \\ y^{*} \rightarrow \bar{y}^{*} \\ \varepsilon \downarrow 0}} \widehat{D}_{\varepsilon}^{*} F(x, y)\left(y^{*}\right), \quad \bar{y}^{*} \in Y^{*}
$$

where $\widehat{D}_{\varepsilon}^{*} F(x, y)$ is defined similarly to (2.2) with the replacement of $N$ by $\widehat{N}_{\varepsilon}$, and where we can equivalently put $\varepsilon=0$ if both spaces $X$ and $Y$ are Asplund and if the graph of $F$ is locally closed around $(\bar{x}, \bar{y})$. As follows from definitions (2.2), (2.3), and (1.2), the only difference between the normal and mixed coderivatives is that the norm convergence of $y^{*} \rightarrow \bar{y}^{*}$ mixed with the weak sequential convergence of $x^{*} \stackrel{w^{*}}{\rightarrow} x^{*}$ are used in (2.3) instead of both weak sequential convergences $y^{*} \stackrel{w^{*}}{\rightarrow} \bar{y}^{*}$ and $x^{*} \stackrel{w^{*}}{\rightarrow} x^{*}$ in the limiting representation of $D_{N}^{*}$. Obviously $D_{M}^{*} F(\bar{x}, \bar{y})\left(y^{*}\right) \subset D_{N}^{*} F(\bar{x}, \bar{y})\left(y^{*}\right)$, where the equality holds if $\operatorname{dim} Y<\infty$. In general, the equality

$$
D_{M}^{*} F(\bar{x}, \bar{y})\left(y^{*}\right)=D_{N}^{*} F(\bar{x}, \bar{y})\left(y^{*}\right), \quad y^{*} \in Y^{*}
$$


is postulated in [16] as the strong coderivative normality of $F$ at $(\bar{x}, \bar{y})$. This property holds for important classes of set-valued and single-valued mappings between infinite-dimensional spaces including convex-graph mappings, the so-called "strictly Lipschitzian" mappings (see below), etc., and it is preserved under various operations; see cases (a)-(i) summarized in [16, Proposition 4.9]. If $F=f: X \rightarrow Y$ is single-valued and strictly differentiable at $\bar{x}$ (which is automatic when $f$ is $C^{1}$ around this point), then

$$
D_{M}^{*} f(\bar{x})\left(y^{*}\right)=D_{N}^{*} f(\bar{x})\left(y^{*}\right)=\left\{\nabla f(\bar{x})^{*} y^{*}\right\}, \quad y^{*} \in Y^{*}
$$

via the adjoint derivative operator $\nabla f(\bar{x})^{*}: Y^{*} \rightarrow X^{*}$. In $[16,17]$, the reader can find equivalent analytic representations of both normal and mixed coderivatives and their efficient calculations for various classes of nonsmooth single-valued and set-valued mappings.

Let $\varphi: X \rightarrow \overline{\mathbb{R}}$ be an extended-real-valued function finite at $\bar{x}$. Then

$$
\partial \varphi(\bar{x}):=\underset{\substack{x \rightarrow \varphi^{\varphi} \bar{x} \\ \varepsilon \downarrow 0}}{\operatorname{Lim} \sup } \widehat{\partial}_{\varepsilon} \varphi(x)
$$

is the (basic, limiting) subdifferential of $\varphi$ at $\bar{x}$, where

$$
\widehat{\partial}_{\varepsilon} \varphi(x):=\left\{x^{*} \in X^{*} \mid \frac{\varphi(u)-\varphi(x)-\left\langle x^{*}, u-x\right\rangle}{\|u-x\|} \geq-\varepsilon\right\}
$$

is the $\varepsilon$-subdifferential of $\varphi$ at $x$, for each $\varepsilon \geq 0$. When $\varepsilon=0$, the set $\hat{\partial} \varphi(x):=\widehat{\partial}_{0} \varphi(x)$ is known also as the presubdifferential, or the Fréchet (regular, viscosity) subdifferential of $\varphi$ at $x$. If $X$ is Asplund and if the function $\varphi$ is lower semicontinuous (l.s.c.) around $\bar{x}$, the sets $\widehat{\partial}_{\varepsilon} \varphi(x)$ can be equivalently replaced by $\widehat{\partial} \varphi(x)$ in (2.4). Furthermore, the subdifferential (2.4) admits the geometric description

$$
\partial \varphi(\bar{x})=\left\{x^{*} \in X^{*} \mid\left(x^{*},-1\right) \in N((\bar{x}, \varphi(\bar{x})) ; \text { epi } \varphi)\right\}
$$

via the normal cone (2.1) to the epigraph epi $\varphi:=\{(x, \mu) \in X \times \mathbb{R} \mid \mu \geq \varphi(x)\}$, useful in the geometric approach to generalized differentiation and applications to optimization. On the other hand, the geometrically defined coderivatives (2.2) and (2.3) admit, in the case of single-valued mappings $f: X \rightarrow Y$, the following representations

$$
D_{M}^{*} f(\bar{x})=\partial\left\langle y^{*}, f\right\rangle(\bar{x}), \quad D_{N}^{*} f(\bar{x})\left(y^{*}\right)=\partial\left\langle y^{*}, f\right\rangle(\bar{x}) \text { as } y^{*} \in Y^{*}
$$

via the basic subdifferential (2.4) of the scalarized function $\left\langle y^{*}, f\right\rangle(x):=\left\langle y^{*}, f(x)\right\rangle$. The first representation in (2.5) holds for every locally Lipschitzian mapping $f$ between Banach spaces, while the second one requires in addition that $X$ is Asplund and that $f$ is strictly Lipschitzian at $\bar{x}$ in the sense that the sequence

$$
\left\{\frac{f\left(x_{k}+t_{k} v\right)-f\left(x_{k}\right)}{t_{k}}\right\}, \quad k \in \mathbb{N}
$$

contains a norm convergent subsequence whenever $x_{k} \rightarrow \bar{x}$ and $v$ belongs to some neighborhood of the origin; see [16, Subsection 3.1.3] for characterizations, verifiable sufficient 
conditions, and applications of the latter property when the space $Y$ is infinite-dimensional (otherwise it obviously reduces the the classical Lipschitz continuity of $f$ around $\bar{x}$ ).

Observe that our basic constructions (2.1)-(2.4) may have nonconvex values even in very simple situations: e.g., $\partial(-|x|)(0)=\{-1,1\}$. It seems surprising therefore, from the viewpoint of conventional techniques in convex analysis totally based on separation theorems, that they enjoy full calculus (mostly in Asplund spaces, although a number of strong and useful results are available in the arbitrary Banach space setting). The main driving force for this calculus and many other results of variational analysis is the fundamental extremal principle (see [16, Chapter 2] for the detailed study and discussions), which is a variational counterpart of convex separation in nonconvex settings.

Next we recall "normal compactness" properties of sets, set-valued mappings, and extended-real-valued functions that are automatic in finite dimensions while playing a crucial role in infinite-dimensional variational analysis and its applications; see [16, 17]. Since these properties are employed in the paper only in the Asplund space setting, we give simplified definitions equivalent to the general ones [16] for the cases under consideration.

A (locally) closed-graph mapping $F: X \rightrightarrows Y$ is sequentially normally compact (SNC) at the point $(\bar{x}, \bar{y}) \in \operatorname{gph} F$ if for any sequences $\left(x_{k}, y_{k}, x_{k}^{*}, y_{k}^{*}\right) \in(\operatorname{gph} F) \times X^{*} \times Y^{*}$ satisfying

$$
\left(x_{k}, y_{k}\right) \rightarrow(\bar{x}, \bar{y}), x_{k}^{*} \in \widehat{D}^{*} F\left(x_{k}, y_{k}\right)\left(y_{k}^{*}\right) \text { and }\left(x_{k}^{*}, y_{k}^{*}\right) \stackrel{w^{*}}{\rightarrow}(0,0)
$$

one has $\left\|\left(x_{k}^{*}, y_{k}^{*}\right)\right\| \rightarrow 0$ as $k \rightarrow \infty$. A set $\Omega$ is $S N C$ at $\bar{x} \in \Omega$ if the constant mapping $F(\cdot) \equiv \Omega$ is SNC at this point. The latter property always holds of $\Omega$ is compactly epiLipschitzian (CEL) around $\bar{x}$ in the sense of Borwein and Strójwas [2] although in general the implication $\mathrm{CEL} \Rightarrow \mathrm{SNC}$ is strict even for convex cones in nonseparable Asplund spaces; see [5] for a comprehensive study of the relationships between the SNC and CEL properties.

A mapping $F: X \Rightarrow Y$ is partially $S N C$ (PSNC) at $(\bar{x}, \bar{y})$ if for any sequences satisfying (2.6) one has $\left\|x_{k}^{*}\right\| \rightarrow 0$ provided that $\left\|y_{k}^{*}\right\| \rightarrow 0$ as $k \rightarrow \infty$. The PSNC property is significantly less restrictive than the SNC one and always holds, in particular, for mappings $F$ having Aubin's Lipschitz-like ("pseudo-Lipschitz") property around $(\bar{x}, \bar{y})$, in the sense that there are neighborhoods $U$ of $\bar{x}$ and $V$ of $\bar{y}$ and a number $\ell \geq 0$ such that

$$
F(x) \cap V \subset F(u)+\ell\|x-u\| \not B \text { whenever } x, u \in U .
$$

When $V=Y$, the latter property reduces to the classical (Hausdorff) Lipschitz continuity of $F$ around $\bar{x}$. Moreover, the simultaneous fulfillment of the PSNC property of $F$ at $(\bar{x}, \bar{y})$ and the mixed coderivative condition

$$
D_{M}^{*} F(\bar{x}, \bar{y})(0)=\{0\}
$$

is necessary and sufficient for $F$ to be Lipschitz-like around $(\bar{x}, \bar{y})$; see [16, Theorem 4.10].

Finally, $F: X \Rightarrow Y$ is strongly $P S N C$ around $(\bar{x}, \bar{y})$ if for any sequences satisfying (2.6) one has $\left\|x_{k}^{*}\right\| \rightarrow 0$ as $k \rightarrow \infty$. This always holds for mappings $F$ partially $C E L$ around $(\bar{x}, \bar{y})$ in the sense of Jourani and Thibault [12].

We refer the reader to $[16,17]$ for other efficient conditions implying the SNC/PSNC properties for specific classes of set-valued and single-valued mappings and to the well- 
developed SNC calculus ensuring the preservation of such properties under various operations; this seems to be the most important for applications. Note that the proofs of the major rules of SNC calculus are also based on the extremal principle.

\section{Multiobjective Optimization and EPECs via Generalized Order Optimality}

In this section we study EPECs, where equilibrium/efficiency relations on the upper level are given by a certain "generalized order optimality" that can be treated from the viewpoint of multiobjective optimization. First we formulate this notion in the vein of [17, Subsection 5.3.1]; see also the references therein.

Definition 3.1 (generalized order optimality under constraints). Given a "cost" mapping $f: X \rightarrow Z$ between Banach spaces, an "ordering" set $\theta \subset Z$ with $0 \in \theta$, and a constraint set $\Omega \subset X$, we say that a point $\vec{x} \in \Omega$ is LOCALLY $(f, \Theta, \Omega)$-OPTIMAL if there are a neighborhood $U$ of $\bar{x}$ and a sequence $\left\{z_{k}\right\} \subset Z$ with $\left\|z_{k}\right\| \rightarrow 0$ as $k \rightarrow \infty$ such that

$$
f(x)-f(\bar{x}) \notin \Theta-z_{k} \text { for all } x \in \Omega \cap U \text { and } k \in \mathbb{N} \text {. }
$$

The set $\theta$ in Definition 3.1 can be viewed as a genierator of an extended order/preference relation between $z_{1}, z_{2} \in Z$ defined by $z_{1}-z_{2} \in \Theta$. In the scalar case of $Z=\mathbb{R}$ and $\Theta=\mathbb{R}_{-}$, the above notion clearly reduces to the standard optimality with the cost function $f$.

Note that we do not assume that the ordering set $\theta$ is either convex or of nonempty interior. If it is a convex subcone of $Z$ with ri $\theta \neq \emptyset$, then the concept of Definition 3.1 encompasses a Pareto-type efficiency/equilibrium requiring that there is no $x \in \Omega \cap U$ with $f(x)-f(\bar{x}) \in \mathrm{ri} \Theta$; to see this, we put $z_{k}:=z_{0} / k, k \in \mathbb{N}$, with some $z_{0} \in \mathrm{ri} \Theta$. The standard weak Pareto efficiency corresponds to the more restrictive relation $f(x)-f(\bar{x}) \in \operatorname{int} \Theta$, while the Pareto efficiency means that there is no $x \in \Omega \cap U$ for which $f(x)-f(\bar{x}) \in \Theta$ and $f(\bar{x})-f(x) \notin \Theta$; compare, e.g., the book by Jahn [11] and its references.

Our goal in this section is to derive necessary optimality conditions for EPECs with equilibrium relations given by the generalized order optimality on the upper level. To begin with, consider the following abstract EPEC given $f: X \times Y \rightarrow Z, 0 \in \Theta \subset Z$, and a set-valued mapping $S: X \Rightarrow Y$,

$$
\text { find a local }(f, \Theta) \text {-optimal point }(\bar{x}, \bar{y}) \text { subject to } y \in S(x) \text {. }
$$

The set-valued mapping $S$ in (3.1) can be viewed as a parametric solution map to abstract constraints of a generalized equilibrium type, which particularly cover those (1.1) of our main interest in this paper. We begin with necessary conditions for local optimal solution to (3.1) in Asplund spaces. For brevity and simplicity, consider only the case when the cost mapping $f$ is locally Lipschitzian; more general cases can be treated in the line of $[17$, Section 5.3]. Note that the primary driving forces for proving the results of this section are the exact extremal principle in product spaces [17] along with the comprehensive generalized differential and SNC calculi developed in [16]. 
Theorem 3.2 (necessary conditions for generalized order optimality in abstract EPECs). Let $(\bar{x}, \bar{y})$ be an optimal solution to (3.1), where $f: X \times Y \rightarrow Z$ is a mapping between Asplund spaces that is locally Lipschitzian around $(\bar{x}, \bar{y})$, and where the sets $\mathrm{gph} S$ and $\Theta$ are locally closed around $\bar{z}:=f(\bar{x}, \bar{y})$ and $0 \in \Theta$, respectively. Assume also that:

(a) either $\Theta$ is SNC at 0 ,

(b) or the inverse mapping $f^{-1}: Z \rightrightarrows X \times Y$ is strongly PSNC at $(\bar{z}, \bar{x} ; \bar{y})$ and

$$
\left[\left(x^{*}, y^{*}\right) \in D_{N}^{*} f(\bar{x}, \bar{y})(0),-x^{*} \in D_{N}^{*} S(\bar{x}, \bar{y})\left(y^{*}\right)\right] \Longrightarrow x^{*}=y^{*}=0
$$

Then there is $z^{*} \in N(0 ; \theta) \backslash\{0\}$ satisfying

$$
0 \in D_{N}^{*} f(\bar{x}, \bar{y})\left(z^{*}\right)+N((\bar{x}, \bar{y}) ; \operatorname{gph} S) .
$$

Moreover, the qualification condition (3.2) is automatic and the necessary optimality condition (3.3) is equivalent to

$$
0 \in \partial\left\langle z^{*}, f\right\rangle(\bar{x}, \bar{y})+N((\bar{x}, \bar{y}) ; \operatorname{gph} S)
$$

provided that $f$ is strictly Lipschitzian at $(\bar{x}, \bar{y})$.

Proof. It is easy to observe that the point $(\vec{x}, \bar{y})$ is locally $(f, \Theta, g p h S)$-optimal in the sense of Definition 3.1 in the product space $X \times Y$. Since both spaces $X$ and $Y$ are assumed to be Asplund, their product $X \times Y$ is also Asplund, by the well-known fact from the theory of Asplund spaces [25]. Thus we can apply the results of [17, Theorem 5.59] that give necessary conditions for general problems of constrained multiobjective optimization. According to assertion (ii) of the latter theorem, whose proof employs the full power of the exact extremal principle in product of Asplund spaces [17, Lemma 5.58] and the corresponding generalized differential and SNC calculi, we find $z^{*} \in N(0 ; \Theta) \backslash\{0\}$ such that

$$
0 \in D_{N}^{*}(f+\Delta(\cdot ; \operatorname{gph} S))(\bar{x}, \bar{y})
$$

provided that either $\Theta$ is SNC at 0 , or the inverse mapping

$$
G(x, y):=[f(x, y)+\Delta((x, y) ; \operatorname{gph} S)]^{-1}
$$

is PSNC at $(\bar{z}, \bar{x}, \bar{y})$. In $(3.5), \Delta(\cdot ; \Omega)$ stands for the indicator mapping of the given set $\Omega \subset W(\Omega=\operatorname{gph} S \subset X \times Y$ in our case) with respect to the image space $Z$ defined by

$$
\Delta(w ; \Omega):= \begin{cases}0 \in W & \text { if } w \in \Omega, \\ \emptyset & \text { if } w \notin \Omega .\end{cases}
$$

Further, we need to present the optimality condition (3.5) and the PSNC assumption on (3.6) in terms of the initial data of the problem (3.1) under consideration.

Applying the coderivative sum rule from [16, Proposition 3.12] and taking into account that the Lipschitz continuity of $f$ around $(\bar{x}, \bar{y})$ yields $D_{M}^{*} f(\bar{x}, \bar{y})(0)=\{0\}$ by $(2.8)$, we conclude that (3.3) follows from (3.5), since

$$
D_{N}^{*} \Delta((\bar{x}, \bar{y}) ; \operatorname{gph} S)\left(z^{*}\right)=N((\bar{x}, \bar{y}) ; \operatorname{gph} S) \text { for all } z^{*} \in Z^{*}
$$


This justifies the necessary optimality condition of the theorem in case (a).

To establish (3.5) in case (b), it remains to show that the the assumptions therein ensure that the mapping $G$ in (3.6) is PSNC at $(\bar{z}, \bar{x}, \bar{y})$. To proceed, we fix arbitrary sequences $\left(x_{k}, y_{k}, x_{k}^{*}, y_{k}^{*}, z_{k}^{*}\right)$ with $\left(x_{k}, y_{k}\right) \rightarrow(\bar{x}, \bar{y})$ satisfying

$$
\left(x_{k}^{*}, y_{k}^{*}\right) \in \widehat{D}^{*}(f+\Delta(\cdot ; \operatorname{gph} S))\left(x_{k}, y_{k}\right)\left(z_{k}^{*}\right), \quad\left\|\left(x_{k}^{*}, y_{k}^{*}\right)\right\| \rightarrow 0, \text { and } z_{k}^{*} \stackrel{w^{*}}{\rightarrow} 0
$$

and show that $\left\|z_{k}^{*}\right\| \rightarrow 0$ as $k \rightarrow \infty$, which means that the required PSNC property of $G$ holds. Observe that the qualification condition (3.2) implies the existence of some sequences $\varepsilon_{k} \downarrow 0,\left(x_{i k}, y_{i k}\right) \rightarrow(\bar{x}, \bar{y})$ for $i=1,2$, and $\left(\tilde{x}_{k}^{*}, \tilde{y}_{k}^{*}, \tilde{z}_{k}^{*}\right)$ satisfying the inclusion

$$
\left(\tilde{x}_{k}^{*}, \tilde{y}_{k}^{*}\right) \in \widehat{D}^{*} f\left(x_{1 k}, y_{1 k}\right)\left(\tilde{z}_{k}^{*}\right)+\widehat{N}\left(\left(x_{2 k}, y_{2 k}\right) ; \operatorname{gph} S\right)
$$

and the estimate

$$
\left\|\left(\tilde{x}_{k}^{*}, \tilde{y}_{k}^{*}, \tilde{z}_{k}^{*}\right)-\left(x_{k}^{*}, y_{k}^{*}, z_{k}^{*}\right)\right\| \leq \varepsilon_{k}
$$

for each $k \in \mathbb{N}$. This can be derived from [16, Lemma 3.1] similarly to the proof of the coderivative sum rule in [16, Theorem 3.10]. Therefore, by (3.7) there exist

$$
\left(x_{1 k}^{*}, y_{1 k}^{*}\right) \in \widehat{D}^{*} f\left(x_{1 k}, y_{1 k}\right)\left(\tilde{z}_{k}^{*}\right) \text { and }\left(x_{2 k}^{*}, y_{2 k}^{*}\right) \in \widehat{N}\left(\left(x_{2 k}, y_{2 k}\right) ; \operatorname{gph} S\right)
$$

ensuring the equalities

$$
\left(\tilde{x}_{k}^{*}, \tilde{y}_{k}^{*}\right)=\left(x_{1 k}^{*}, y_{1 k}^{*}\right)+\left(x_{2 k}^{*}, y_{2 k}^{*}\right), \quad k \in \mathbb{N}
$$

It follows from $z_{k}^{*} \stackrel{w^{*}}{\rightarrow} 0$ and estimate (3.8) with respect to the $z$-component that $\tilde{z}_{k}^{*} \stackrel{w^{*}}{\rightarrow} 0$ as $k \rightarrow \infty$. Hence, by the classical uniform boundedness principle, the sequence $\left\{\tilde{z}_{k}^{*}\right\}$ is bounded in $Z^{*}$. Since $f$ is locally Lipschitzian around $(\bar{x}, \bar{y})$ with some modulus $\ell \geq 0$, the first inclusion in (3.9) implies by [16, Theorem 1.43] that

$$
\left\|\left(x_{1 k}^{*}, y_{1 k}^{*}\right)\right\| \leq \ell\left\|\tilde{z}_{k}^{*}\right\| \text { for all } k \in \mathbb{N}
$$

which ensures the boundedness of the sequence $\left\{\left(x_{1 k}^{*}, y_{1 k}^{*}\right)\right\}$ in $X^{*} \times Y^{*}$. Thus, by the sequential weak* compactness of bounded sets in dual to Asplund spaces [25], the latter sequence contains a subsequence weak* converging to some $\left(x^{*}, y^{*}\right) \in X^{*} \times Y^{*}$. It follows from the first inclusion in (3.9) with $\tilde{z}_{k}^{*} \stackrel{w^{*}}{\rightarrow} 0$ and from definition (2.2) of the normal coderivative that $\left(x^{*}, y^{*}\right) \in D_{N}^{*} f(\bar{x}, \bar{y})(0)$. We similarly conclude from (3.8) and (3.10) that $\left\{\left(x_{2 k}^{*}, y_{2 k}^{*}\right)\right\}$ weak* converges to some $\left(x_{2}^{*}, y_{2}^{*}\right) \in X^{*} \times Y^{*}$ along a subsequence and that actually $\left(x_{2}^{*}, y_{2}^{*}\right) \in N((\bar{x}, \bar{y}) ;$ gph $S)$ due to $(2.1)$ by passing to the limit in the second inclusion of (3.9). Moreover, $\left(x_{2}^{*}, y_{2}^{*}\right)=\left(-x^{*},-y^{*}\right)$ by passing to the limit in equality (3.10). Thus

$$
\left(x^{*} ; y^{*}\right) \in D_{N}^{*} f(\bar{x}, \bar{y})(0) \cap[-N((\bar{x}, \bar{y}) ; \operatorname{gph} S)]
$$

and hence $\left(x^{*}, y^{*}\right)=(0,0)$ by the qualification condition (3.2). The latter implies that

$$
\left(x_{1 k}^{*}, y_{1 k}^{*}, z_{k}^{*}\right) \stackrel{w^{*}}{\rightarrow} 0 \text { with }\left(x_{1 k}^{*}, y_{1 k}^{*}\right) \in \widehat{D}^{*} f\left(x_{1 k}, y_{1 k}\right)\left(z_{k}^{*}\right), \quad k \in \mathbb{N} .
$$


Invoking now the strong PSNC property of $f^{-1}$ at $(\bar{z}, \bar{x}, \bar{y})$, we conclude that $\left\|z_{k}^{*}\right\| \rightarrow 0$ as $k \rightarrow \infty$, which justifies the required PSNC property of $G$ in (3.6) and thus the optimality condition (3.3) under the assumptions in (b).

Suppose finally that $f$ is strictly Lipschitzian at $(\bar{x}, \bar{y})$. Then

$$
D_{N}^{*} f(\bar{x}, \bar{y})\left(z^{*}\right)=\partial\left\langle z^{*}, f\right\rangle(\bar{x}, \bar{y}) \text { for all } z^{*} \in Z^{*}
$$

by the scalarization formula of [16, Theorem 3.28]. Hence (3.3) is equivalent to (3.4) in this case and the qualification condition (3.2) trivially holds due to $D_{N}^{*} f(\bar{x}, \bar{y})(0)=\{0\}$. This completes the proof of the theorem.

Observe that the necessary conditions of Theorem 3.2 are established in the normal form, with a nonzero "multiplier" $z^{*}$ corresponding to the cost mapping $f$. This is due to the imposed qualification condition (3.2). Since the optimality and qualification conditions obtained are of the same (dual) nature, they can be unified in a single necessary optimality condition written in the non-qualified form, which ensures the nontriviality of the whole collection of "multipliers" corresponding to the cost mapping and constraints, while does not exclude that the "cost multiplier" equals zero.

Corollary 3.3 (non-qualified necessary conditions for abstract EPECs). Assume that, in the general framework of Theorem 3.2, either $\Theta$ is SNC at 0 or $f^{-1}$ is strongly PSNC at $(\bar{z}, \bar{x}, \bar{y})$. Then there are $0 \neq\left(x^{*}, y^{*}, z^{*}\right) \in X^{*} \times Y^{*} \times Z^{*}$ satisfying

$$
\left(x^{*}, y^{*}\right) \in D_{N}^{*} f(\bar{x}, \bar{y})\left(z^{*}\right), \quad-x^{*} \in D_{N}^{*} S(\bar{x}, \bar{y})\left(y^{*}\right), \quad z^{*} \in N(0 ; \theta) .
$$

Proof. When $\Theta$ is SNC at $0,(3.11)$ is the same as (3.3) with $z^{*} \in N(0 ; \Theta) \backslash\{0\}$ by the normal coderivative definition (2.2). When $f^{-1}$ is strongly PSNC at $(\bar{z}, \bar{x}, \bar{y})$ and the qualification condition (3.2) is satisfied, (3.11) also reduces to (3.3) with $z^{*} \in N(0 ; \Theta) \backslash\{0\}$. On the other hand, the negation of (3.2) means that (3.11) holds with some $\left(x^{*}, y^{*}\right) \neq 0$.

Next let us consider our main problem in this section when the mapping $S(\cdot)$ in the abstract EPEC (3.1) is given in the form of equilibrium constraints (1.1), i.e., $S(\cdot)$ is the solution map to the parametric generalized equations/variational systems

$$
S(x):=\{y \in Y \mid 0 \in q(x, y)+Q(x, y)\}
$$

For brevity and simplicity, we restrict ourselves to the case of strictly Lipschitzian singlevalued mappings under consideration. These assumptions can be dropped or significantly relaxed on the base of Theorem 3.2 and appropriate rules of generalized differential and SNC calculi (cf. [17]); however, it leads us to results technically more involved and complicated in formulation. The result formulated in the theorem is given in the general non-qualified form, which can be equivalently formulated in the corresponding normal form under the so-called Fredholm qualification condition. Note also that we consider in (3.12) the case of parameter-dependent multivalued fields $Q=Q(x, y)$ of generalized equations, in contrast to the majority of other studies in this direction. 
Theorem 3.4 (necessary conditions for EPECs governed by parameter-dependent generalized equations). Let $(\bar{x}, \bar{y})$ be a solution to (3.1) with $S(\cdot)$ given by (3.12), where $f: X \times Y \rightarrow Z, q: X \times Y \rightarrow P$, and $Q: X \times Y \Rightarrow P$ are mappings between Asplund spaces, and where $\Theta \subset Z$ with $0 \in \Theta$. Assume that $f$ and $q$ are strictly Lipschitzian at $(\bar{x}, \bar{y})$, that $\Theta$ is locally closed around the origin, and that $Q$ is locally closed around $(\bar{x}, \bar{y}, \bar{p})$ with $\bar{p}:=-q(\bar{x}, \bar{y})$. Then there are $\left(z^{*}, p^{*}\right) \in Z^{*} \times P^{*}$, not both zero, such that

$$
0 \in \partial\left\langle z^{*}, f\right\rangle(\bar{x}, \bar{y})+\partial\left\langle p^{*}, q\right\rangle(\bar{x}, \bar{y})+D_{N}^{*} Q(\bar{x}, \bar{y}, \bar{p})\left(p^{*}\right) \text { and } z^{*} \in N(0 ; \Theta)
$$

in each of the following cases:

(a) $\Theta$ is $S N C$ at 0 ;

(b) $f^{-1}$ is strongly PSNC at $(\bar{z}, \bar{x}, \bar{y})$ with $\bar{z}:=f(\bar{x}, \bar{y})$ and either $Q$ is SNC at $(\bar{x}, \bar{y}, \bar{p})$, or $\operatorname{dim} P<\infty$.

Proof. We are based on the assumptions and results of Corollary 3.3, where

$$
\left(x^{*}, y^{*}\right) \in D_{N}^{*} f(\bar{x}, \bar{y})\left(z^{*}\right) \Longleftrightarrow\left(x^{*}, y^{*}\right) \in \partial\left\langle z^{*}, f\right\rangle(\bar{x}, \bar{y})
$$

due to the strict Lipschitzian requirement on $f$ at $(\tilde{x}, \bar{y})$. Thus, under the latter assumption in addition to the other assumptions of Corollary 3.3, it ensures the existence of $\left(x^{*}, y^{*}, z^{*}\right)$ satisfying (3.11) with $z^{*} \neq 0$.

Let us now express/estimate the normal coderivative $D_{N}^{*} S(\bar{x}, \bar{y})$ for the mapping $S$ in (3.12) via the initial data of (3.12) and the requirements imposed directly on $q$ and $Q$. Employing [16, Theorem 4.46], we get the upper estimate

$$
\begin{aligned}
D_{N}^{*} S(\bar{x}, \bar{y})\left(y^{*}\right) \subset\{ & \left\{x^{*} \in X^{*} \mid \exists p^{*} \in P^{*}\right. \text { with } \\
& \left.\left(x^{*},-y^{*}\right) \in \partial\left\langle p^{*}, q\right\rangle(\bar{x}, \bar{y})+D_{N}^{*} Q(\bar{x}, \bar{y}, \bar{p})\left(p^{*}\right)\right\}
\end{aligned}
$$

provided that the adjoint generalized equation to (1.1) has only the trivial solution:

$$
\left[0 \in \partial\left\langle p^{*}, q\right\rangle(\bar{x}, \bar{y})+D_{N}^{*} Q(\bar{x}, \bar{y}, \bar{p})\left(p^{*}\right)\right] \Longrightarrow p^{*}=0
$$

and that either $Q$ is SNC at $(\bar{x}, \bar{y}, \bar{p})$, or $\operatorname{dim} P<\infty$. Substituting (3.14) and (3.15) into (3.11), we arrive at (3.13) with $z^{*} \neq 0$ under the qualification condition (3.16) and the assumptions made in the theorem.

On the other hand, the negation of $(3.16)$ means that there is $0 \neq p^{*} \in P^{*}$ satisfying

$$
0 \in \partial\left\langle p^{*}, q\right\rangle(\bar{x}, \bar{y})+D_{N}^{*} Q(\bar{x}, \bar{y}, \bar{p})\left(p^{*}\right)
$$

This gives (3.13) with $z^{*}=0$ and $p^{*} \neq 0$, which completes the proof of the theorem.

The qualification condition (3.16) and its counterparts for more general mappings $q$ in (1.1) play a significant role in the analysis of equilibrium constraints (sensitivity, optimality conditions for MPECs and EPECs, etc.); see [16, 17]. It reminds us Fredholm's alternative for integral equations, where the triviality of solutions to the adjoint equation is a crucial condition for solvability of the original one. For this reason, we call $(3.16)$ the Fredholm 
qualification condition for generalized equations and the associated MPECs and EPECs. As follows from the proof of Theorem 3.4, the qualification assumption (3.16) ensures the normal form of the necessary conditions in (3.13) with $z^{*} \neq 0$.

Let us next present further elaborations of the results obtained for some important classes of EPECs, where the multivalued part (field) of the equilibrium constraints (1.1) is given in a subdifferential form typical for the majority of applications. Usually subdifferential structures arising in applications involve certain compositions. . We pay the main attention to the composite subdifferential structures given in the following forms:

$$
Q(x, y)=\partial(\psi \circ g)(x, y) \text { or } Q(x, y)=(\partial \psi \circ g)(x, y)
$$

where $g$ is a single-valued mapping between Banach spaces and where $\psi$ is an extendedreal-valued function. For convenience, we refer (borrowing mechanical terminology) to the first structure in (3.17) as to that with composite potentials, while the second structure the second structure in (3.17) is that with composite fields. The subdifferential in (3.17) is taken in our basic sense (2.4), while other subdifferential constructions can be considered as well in a similar way.; compare, e.g., [18]. Parametric generalized equations (equilibrium constraints) with multivalued parts given in both forms (3.17) encompass a variety of parametric variational systems including particularly variational inequalities and nonlinear complementarity (as well as implicit complementarity) problems, sets of stationary solutions in nonlinear programming, hemivariational and quasivariational inequalities, etc.; see more discussions and examples in the books $[6,13,16,24]$ and the references therein.

Observe that equilibrium constraints (1.1) with subdifferential structures (3.17) contain by construction a first-order information arising, in particular, from first-order necessary conditions in lower-level optimization problems. Thus necessary conditions (and related results) for upper-level problems with equilibrium constraints of the (first-order) subdifferential type naturally require certain second-order generalized differential objects.

Recall the second-order subdifferential notion for extended-real-valued functions used in what follows; see the book [16] and its references for more details and historical comments. Given $\varphi: X \rightarrow \overline{\mathbb{R}}$ finite at $\bar{x}$ and given $\bar{y} \in \partial \varphi(\bar{x})$, the (normal) second-order subdifferential of $\varphi$ at $\bar{x}$ relative to $\bar{y}$ is defined by

$$
\partial^{2} \varphi(\bar{x}, \bar{y})(u):=\left(D_{N}^{*} \partial \varphi\right)(\bar{x}, \bar{y})(u), \quad u \in X^{* *},
$$

i.e., as the (normal) coderivative (2.2) of the first-order subdifferential mapping (2.4). When $\varphi \in C^{2}$ around $\bar{x}$, the set (3.18) is a singleton for each $u \in X^{* *}$ reducing to the classical second-order derivative (Hessian) of $\varphi$ at $\bar{x}$ :

$$
\partial^{2} \varphi(\bar{x})(u)=\left\{\nabla^{2} \varphi(\bar{x})^{*} u\right\}, \quad u \in X^{* *},
$$

where the adjoint operation is not needed in finite-dimensional spaces, due to the symmetricity of the classical Hessian matrix. In general, (3.18) defines a positively homogeneous set-valued mapping from $X^{* *}$ into $X^{*}$, which possesses an extensive calculus in both finite and infinite dimensions; see [16]. Besides various situations and examples considered in the books $[16,17]$ and the references therein, we particularly refer the reader to the papers 
$[4,18,19]$ containing precise calculations of the second-order subdifferential for favorable classes of extended-real-valued functions arising in various optimization and equilibrium problems motivated by numerous applications.

Let us first consider EPECs whose equilibrium constraints are governed by the subdifferential generalized equations with composite potentials

$$
0 \in q(x, y)+\partial(\psi \circ g)(x, y)
$$

where $q: X \times Y \rightarrow X^{*} \times Y^{*}, g: X \times Y \rightarrow W$, and $\psi: W \rightarrow \overline{\mathbb{R}}$. The following two theorems distinguish between problems with parameter-independent fields when $g=g(y)$ in (3.19), and those with $g=g(x, y)$. Although the second framework obviously includes the first one, the results presented below are independent of each other, since in the first case we are able to work with general spaces, due to the available calculus. As before, we restrict our consideration to EPECs with strictly Lipschitzian costs. Note that the closedgraph assumption (in the norm topology of $W \times W^{*}$ ) on the subdifferential mapping $\partial \psi$ imposed in the next and subsequent results of this section is automatic if either $\psi$ is locally continuous, or it is amenable at the reference point; see below.

Theorem 3.5 (necessary conditions for EPECs with parameter-independent potentials). Let $(\bar{x}, \bar{y})$ be a solution to the EPEC given in (3.i) with

$$
S(x):=\{y \in Y \mid 0 \in q(x, y)+\partial(\psi \circ g)(y)\}
$$

where $f: X \times Y \rightarrow Z$ is strictly Lipschitzian at $(\bar{x}, \bar{y})$, where $\Theta \subset Z$ is locally closed around $0 \in \Theta$, where $g$ is strictly differentiable at $(\bar{x}, \bar{y})$ with the surjective partial derivative $\nabla_{x} q(\bar{x}, \bar{y})$, and where $g=g(y) \in C^{2}$ around $\bar{y}$ with the surjective derivative $\nabla g(\bar{y})$. Assume also that the spaces $X$ and $Z$ are Asplund while $\operatorname{dim} Y<\infty$ and $W$ is Banach, and that the graph of the subdifferential mapping $\partial \psi$ is locally closed around $(\bar{w}, \bar{v})$ with $\bar{w}:=g(\bar{y})$ and $\bar{v}$ being a unique solution to the system

$$
-q(\bar{x}, \bar{y})=\nabla g(\bar{y})^{*} \bar{v}, \quad \bar{v} \in \partial \psi(\bar{w}) .
$$

Then there are $z^{*} \in N(0 ; \theta) \backslash\{0\}$ and $u \in Y$ satisfying

$$
\begin{aligned}
0 \in \quad & \partial\left\langle z^{*}, f\right\rangle(\bar{x}, \bar{y})+\nabla q(\bar{x}, \bar{y})^{*} u \\
& +\left(0, \nabla^{2}\langle\bar{v}, g\rangle(\bar{y})^{*} u+\nabla g(\bar{y})^{*} \partial^{2} \psi(\bar{w}, \bar{v})(\nabla g(\bar{y}) u)\right)
\end{aligned}
$$

provided that either $\Theta$ is SNC at 0 , or $f^{-1}$ is strongly PSNC at $(\bar{z}, \bar{x}, \bar{y})$ with $\bar{z}:=f(\bar{x}, \bar{y})$.

Proof. We are based on Theorem 3.4, where $P=Y^{*}\left(=\mathbb{R}^{m}\right), Q(y)=\partial(\psi \circ g)(y)$, and

$$
\partial\langle u, q\rangle(\bar{x}, \bar{y})=\nabla q(\bar{x}, \bar{y})^{*} u, \quad u \in Y
$$

due to the assumed strict differentiability of $q$ at $(\bar{x}, \bar{y})$. Observe that $Q=\partial(\psi \circ g)$ is locally closed-graph by the assumptions on $\partial \psi$ and $g$. Since the partial derivative $\nabla_{x} q(\bar{x}, \bar{y})$ is surjective and $Q=Q(y)$, the Fredholm qualification condition (3.16) is fulfilled. Thus the 
necessary optimality conditions in (3.13) hold in the normal form, i.e., with $z^{*} \neq 0$. We need to compute

$$
D_{N}^{*} Q(\bar{y}, \bar{p})(u)=\partial^{2}(\psi \circ g)(\bar{y}, \bar{p})(u) \text { with } \bar{p}:=-q(\bar{x}, \bar{y}) .
$$

Employing the second-order subdifferential chain rule from [16, Theorem 1.127] held in general Banach spaces under the surjectivity assumption on $\nabla g(\bar{y})$ for the mapping $g \in C^{2}$, we get the equality

$$
\partial^{2}(\psi \circ g)(u)=\nabla^{2}\langle\bar{v}, g\rangle(\bar{y})^{*} u+\nabla g(\bar{y})^{*} \partial^{2} \psi(\bar{w}, \bar{v})(\nabla g(\bar{y}) u)
$$

where $\bar{v}$ is uniquely determined by (3.20). Substituting this into (3.13) with $Q=Q(y)$ and taking into account that $P=Y^{*}$ is finite-dimensional, we arrive at (3.21) under the assumptions made and thus complete the proof of the theorem.

The next result concerns EPECs (3.1) governed by parameter-dependent equilibrium constraints in the subdifferential form with composite potentials (3.19). In contrast to the preceding theorem, we consider the case when all the spaces involved but the image space $Z$ for the cost mapping are finite-dimensional. At the same time, the structure of the composite potential $\psi \circ g$ is significantly more general than in Theorem 3.5: besides the parameter-dependence, we allow $\nabla g(\bar{x}, \bar{y})$ to be non-surjective. More precisely, we consider the so-called strongly amenable potentials $\psi \circ g$, where $\psi$ is l.s.c. and convex while $g$ is $C^{2}$ around the reference points under the first-order qualification condition

$$
\partial^{\infty} \psi(\bar{w}) \cap \operatorname{ker} \nabla g(\bar{x}, \bar{y})^{*}=\{0\} \text { with } \bar{w}:=g(\bar{x}, \dot{y}) ;
$$

see [30] and also [16] for more details concerning this remarkable class of functions largely encountered in finite-dimensional variational analysis and parametric optimization. In (3.22),

$$
\partial \psi(\bar{w}):=\left\{w^{*} \in W^{*} \mid\left(w^{*},-1\right) \in N((\bar{w}, \psi(\bar{w})) ; \operatorname{gph} \psi)\right\}
$$

stands for the singular subdifferential of $\psi$ at $\bar{w}$, which reduces to the singleton $\{0\}$ if $\psi$ is locally Lipschitzian around $\bar{w}$.

Theorem 3.6 (necessary conditions for EPECs with parameter-dependent amenable potentials). Let $(\bar{x}, \bar{y})$ be a solution to the EPEC given in (3.1) with

$$
S(x):=\left\{y \in \mathbb{R}^{m} \mid 0 \in q(x, y)+\partial(\psi \circ g)(y)\right\},
$$

where $f: \mathbb{R}^{n} \times \mathbb{R}^{m} \rightarrow Z$ is strictly Lipschitzian at $(\bar{x}, \bar{y})$, where $Z$ is Asplund and $\Theta \subset Z$ is locally closed around $0 \in \Theta$, where $q: \mathbb{R}^{n} \times \mathbb{R}^{m} \rightarrow \mathbb{R}^{n} \times \mathbb{R}^{m}$ is locally Lipschitzian around $(\bar{x}, \bar{y})$, and where the potential $\psi \circ g$ is strongly amenable at this point with $g: \mathbb{R}^{n} \times \mathbb{R}^{m} \rightarrow \mathbb{R}^{l}$, $\bar{w}:=g(\bar{x}, \bar{y})$. Denote

$$
M(\bar{x}, \bar{y}):=\left\{\bar{v} \in \mathbb{R}^{l} \mid \bar{v} \in \partial \psi(\bar{w}), \nabla g(\bar{x}, \bar{y})^{*} \bar{v}=-q(\bar{x}, \bar{y})\right\}
$$

and impose the second-order qualification condition:

$$
\partial^{2} \psi(\bar{w}, \bar{v})(0) \cap \operatorname{ker} \nabla g(\bar{x}, \bar{y})^{*}=\{0\} \text { for all } \bar{v} \in M(\bar{x}, \bar{y}) .
$$


Then there are $z^{*} \in N(0 ; \Theta)$ and $u \in \mathbb{R}^{n} \times \mathbb{R}^{m}$, not both zero, such that

$$
\begin{aligned}
0 \in \partial\left\langle z^{*}, f\right\rangle(\bar{x}, \bar{y})+\partial\langle u, q\rangle(\bar{x}, \bar{y})+ & \bigcup_{\bar{v} \in M(\bar{x}, \bar{y})}\left[\nabla^{2}\langle\bar{v}, g\rangle(\bar{x}, \bar{y})(u)\right. \\
& \left.+\nabla g(\bar{x}, \bar{y})^{*} \partial^{2} \psi(\bar{w}, \bar{v})(\nabla g(\bar{x}, \bar{y}) u)\right]
\end{aligned}
$$

provided that either $\Theta$ is SNC at 0 , or $f^{-1}$ is strongly PSNC at $(\bar{z}, \bar{x}, \bar{y})$ with $\bar{z}:=f(\bar{x}, \bar{y})$.

Proof. We are now based on Theorem 3.4, where $P=\mathbb{R}^{n} \times \mathbb{R}^{m}$ and where

$$
Q(x, y)=\partial(\psi \circ g)(x, y)
$$

To proceed, we employ the second-order chain rule from [16, Corollary 3.76] involving strongly amenable functions under the assumptions made. Then the second-order qualification condition (3.23) ensures that

$$
\partial^{2}(\psi \circ g)(\bar{x}, \bar{y})(u) \subset \bigcup_{\bar{v} \in M(\bar{x}, \bar{y})}\left[\nabla^{2}\langle\bar{v}, g\rangle(\bar{x}, \bar{y})(u)+\nabla g(\bar{x}, \bar{y})^{*} \partial^{2} \psi(\bar{w}, \bar{v})(\nabla g(\bar{x}, \bar{y}) u)\right]
$$

for all $u \in \mathbb{R}^{n} \times \mathbb{R}^{m}$. Substituting (3.24) into (3.13), we arrive at the necessary optimality conditions of the theorem for the EPEC under consideration.

Observe that the second-order qualification condition (3.23) automatically holds when either $\psi \in C^{1,1}$ around $\bar{w}$ (i.e., it is $C^{1}$ with the local Lipschitzian derivative $\nabla \psi$ ), or the derivative $\nabla g(\bar{x}, \bar{y})$ is surjective. In general, none of these assumptions is required.

Finally in this section, consider EPECs whose equilibrium constraints are governed by the subdifferential generalized equations with composite fields

$$
0 \in q(x, y)+(\partial \psi \circ g)(x, y)
$$

where $g: X \times Y \rightarrow W, \psi: W \rightarrow \overline{\mathbb{R}}$, and $q: X \times Y \rightarrow W^{*}$.

Theorem 3.7 (necessary conditions for EPECs with general composite fields), Let $(\bar{x}, \bar{y})$ be a solution to the EPEC given in (3.1) with

$$
S(x):=\{y \in Y \mid 0 \in q(x, y)+(\partial \psi \circ g)(x, y)\}
$$

where $f: X \times Y \rightarrow Z$ is a mapping between Asplund spaces that is strictly Lipschitzian at $(\bar{x}, \bar{y})$, where $\Theta \subset Z$ is locally. closed around $0 \in \Theta$, where the mappings $q: X \times Y \rightarrow \mathbb{R}^{l}$ and $g: X \times Y \rightarrow \mathbb{R}^{l}$ are locally Lipschitzian around $(\bar{x}, \bar{y})$, and where the graph of $\partial \psi$ is locally closed around $(\bar{w}, \bar{p})$ with $\bar{w}:=g(\bar{x}, \bar{y})$ and $\bar{p}:=-q(\bar{x}, \bar{y})$; the latter is automatic when $\psi: \mathbb{R}^{l} \rightarrow \overline{\mathbb{R}}$ is either amenable at $\bar{w}$ or continuous around this point. Impose also the second-onder qualification condition

$$
\partial^{2} \psi(\bar{w}, \bar{p})(0) \cap\left\{v \in \mathbb{R}^{l} \mid 0 \in \partial\langle v, g\rangle(\bar{x}, \bar{y})\right\}=\{0\} .
$$

Then there are $z^{*} \in N(0 ; \Theta)$ and $u \in \mathbb{R}^{l}$, not both zero, such that

$$
0 \in \partial\left\langle z^{*}, f\right\rangle(\bar{x}, \bar{y})+\partial\langle u, q\rangle(\bar{x}, \bar{y})+\left\{\partial\langle v, g\rangle(\bar{x}, \bar{y}) \mid v \in \partial^{2} \psi(\bar{w}, \bar{p})(u)\right\}
$$

provided that either $\Theta$ is SNC at 0 , or $f^{-1}$ is strongly PSNC at $(\bar{z}, \bar{x}, \bar{y})$. 
Proof. Again we are based on Theorem 3.4, where $P=\mathbb{R}^{l}$ and where

$$
Q(x, y)=(\partial \psi \circ g)(x, y)
$$

To apply the necessary condition (3.13) of the latter theorem, we need to express the coderivative $D^{*}=D_{N}^{*}$ of the composition $\partial \psi \circ g$ with values in $\mathbb{R}^{l}$ via the generalized differential constructions for $\psi$ and $g$ at the corresponding points. The appropriate coderivative chain rule of [16, Theorem 3.13], the scalarization formula of [16, Theorem 3.28], and construction (3.18) of the second-order subdifferential yield the upper estimate

$$
D^{*}(\partial \psi \circ g)(\bar{x}, \bar{y}, \bar{p})(u) \subset\left\{\partial\langle v, g\rangle(\bar{x}, \bar{y}) \mid v \in \partial^{2} \psi(\bar{w}, \bar{p})(u)\right\}
$$

under the second-order qualification condition (3.25). Substituting (3.27) into (3.13), we arrive at the necessary optimality condition of the theorem.

If the inner mapping $g$ in the equilibrium constraint composition happens to be strictly differentiable at $(\bar{x}, \bar{y})$, the results of Theorem 3.7 admit significant simplifications.

Corollary 3.8 (necessary conditions for EPECs with composite fields of special structure). Suppose that in the framework of Theorem 3.7 the inner composite mapping $g: X \times Y \rightarrow \mathbb{R}^{l}$ is strictly differentiable at $(\bar{x}, \bar{y})$. Then all the conclusions of this theorem hold with the replacement of the qualification condition (3.25) and the necessary optimality condition (3.27) by, respectively, (3.23) and

$$
0 \in \partial\left\langle z^{*}, f\right\rangle(\bar{x}, \bar{y})+\partial\langle u, q\rangle(\bar{x}, \bar{y})+\nabla g(\bar{x}, \bar{y})^{*} \partial^{2} \psi(\bar{w}, \bar{p})(u)
$$

Proof. It follows from the subdifferential representation

$$
\partial\langle v, g\rangle(\bar{x}, \bar{y})=\left\{\nabla g(\bar{x}, \bar{y})^{*} v\right\}
$$

held for strictly differentiable mappings.

\section{Multiobjective Optimization and EPECs with Closed Pref- erence Relations}

The main objective of this section is to study EPECs whose preference/equilibrium relations on the upper level are defined via the so-called closed preferences. The results obtained in this ways are generally independent of those in Section 3.

Given a Banach space $Z$ and a subset $R \subset Z \times Z$, we say that $z_{1}$ is preferred to $z_{2}$ (notation $\left.z_{1} \prec z_{2}\right)$ if $\left(z_{1}, z_{2}\right) \in R$. In what follows, we consider nonreflexive preference relations, i.e., such that the preference set $R$ does not contain the diagonal.

Definition 4.1 (closed preference relations). Let

$$
\mathcal{L}(z):=\{u \in Z \mid u \prec z\}
$$


be a LEVEL SET at $z \in Z$ with respect to the given preference $\prec$. We say that $\prec$ is LOCALLY SATIATED around $\bar{z}$ if $z \in \mathrm{cl} \mathcal{L}(z)$ for all $z$ in some neighborhood of $\bar{z}$. Furthermore, the preference $\prec$ is ALMOST TRANSITIVE on $Z$ provided that for all $u \prec z$ and $v \in \operatorname{cl} \mathcal{L}(u)$ one has $v \prec z$. The preference relation $\prec$ is called CLOSED around $\bar{z}$ if it is locally satiated and almost transitive simultaneously.

Observe that the main difference between the preference concepts from Definition 4.1 and Definition 3.1 is the following: instead of the linear translation of sets in the extremal system induced by generalized order optimality, preference relations of Definition 4.1 involve nonlinear transformations. The reader can find detailed discussions and references in [17, Subsection 5.3.1], which particularly show that the two concepts are generally independent. It happens that the main restriction in Definition 4.1 is the almost transitivity requirement on the preference $\prec$, which does not hold for a number of preferences important in applications (e.g., for the lexicographical order), while Definition 3.1 is applied.

To proceed in deriving necessary conditions for EPECs with closed preference relations on the upper equilibrium level, we recall two generalized differential and normal compactness notions for moving sets needed in what follows. Since the results presented below concern closed-graph mappings between Asplund spaces, we give simplified versions of these notions equivalent to the general ones ([17, Subsection 5.3.3] in the settings under consideration.

Definition 4.2 (extended normals and sequential normal compactness for moving sets). Let $\Omega: Z \Rightarrow X$ with $(\bar{z}, \bar{x}) \in \operatorname{gph} \Omega$. Then:

(i) The conic set

$$
N_{+}(\bar{x} ; \Omega(\bar{z})):=\operatorname{Limsup}_{(z, x) \rightarrow(\bar{z}, \bar{x})} \widehat{N}(x ; \Omega(z))
$$

is called the EXTENDED NORMAL CONE to $\Omega(\bar{z})$ at $\bar{x}$.

(ii) The mapping $\Omega(:)$ is IMAGELY SNC (or just ISNC) at $(\bar{z}, \bar{x}) \in \operatorname{gph} \Omega$ if for any sequences $\left(z_{k}, x_{k}, x_{k}^{*}\right)$ satisfying

$$
x_{k}^{*} \in \hat{N}\left(x_{k} ; \Omega\left(z_{k}\right)\right), \quad\left(z_{k}, x_{k}\right) \stackrel{\operatorname{gph} \Omega}{\rightarrow}(\bar{z}, \bar{x}), \quad \text { and } \quad x_{k}^{*} \stackrel{w^{*}}{\rightarrow} 0
$$

one has $\left\|x_{k}^{*}\right\| \rightarrow 0$ as $k \rightarrow \infty$.

Observe that we always have the inclusion (in the setting under consideration)

$$
N(\bar{x} ; \Omega(\bar{z})) \subset N_{+}(\bar{x} ; \Omega(\bar{z}))
$$

where the equality holds under the so-called normal semicontinuity of $\Omega$ at $(\bar{z}, \bar{x})$, which is the case for a broad class of mappings under reasonable assumptions; see [17, Subsection 5.3.3] for more discussions and sufficient conditions.

The ISNC property is obviously automatic in finite dimensions, while in infinite dimensions it holds under certain uniform Lipschitz-type assumptions; see the above reference and [21] for precise results and discussions. Note also that full calculus is available for the ISNC property of moving sets, similarly to that for "non-moving" objects. 
Consider first an abstract $E P E C$, where equilibrium relations on the upper level are described by an arbitrary closed preference $\prec$, while equilibrium constraints are formalize via a general set-valued mapping $S(\cdot)$. Given $f: X \times Y \rightarrow Z$ and $S: X \Rightarrow Y$, a local optimal solution $(\bar{x}, \bar{y}) \in \operatorname{gph} S$ to the following problem is understood as such a feasible point that $f(\bar{x}, \bar{y})$ is not preferred to $f(x, y)$, with respect to the given closed preference $\prec$ on $Z$, to any other feasible point $(x, y) \in \operatorname{gph} S$ around $(\bar{x}, \bar{y})$ :

find a local optimal solution to $f(x, y)$ with respect to $\prec$ subject to $y \in S(x)$.

Theorem 4.3 (necessary conditions for abstract EPECs with closed preference relations). Let $(\bar{x}, \bar{y})$ be a solution to the abstract EPEC given in (4.2), where $\prec$ is a closed preference on $Z$ with the level set $\mathcal{L}(\cdot)$, where $f: X \times Y \rightarrow Z$ is a mapping between Asplund spaces that is locally continuous around $(\bar{x}, \bar{y})$ with $\bar{z}:=f(\bar{x}, \bar{y})$, and where $S: X \Rightarrow Y$ is closed-graph around $(\bar{x}, \bar{y})$. Then there is a nonzero triple $\left(x^{*}, y^{*}, z^{*}\right) \in X^{*} \times Y^{*} \times Z^{*}$ satisfying the relationships

$$
\left(x^{*}, y^{*}\right) \in D_{N}^{*} f(\bar{x}, \bar{y})\left(z^{*}\right), \quad-x^{*} \in D_{N}^{*} S(\bar{x}, \bar{y})\left(y^{*}\right), \text { and } z^{*} \in N_{+}(\bar{z} ; \operatorname{cl} \mathcal{L}(\bar{z}))
$$

provided that either $f$ is $S N C$ at $(\bar{x}, \bar{y})$, or $S$ is $S N C$ at $(\bar{x}, \bar{y})$ and $\operatorname{cl} \mathcal{L}: Z \Rightarrow Z$ is ISNC at $(\bar{z}, \bar{z})$. Furthermore, (4.3) with $\left(x^{*}, y^{*}, z^{*}\right) \neq 0$ is equivalent to

$$
0 \in \partial\left\langle z^{*}, f\right\rangle(\bar{x}, \bar{y})+N((\bar{x}, \bar{y}) ; \operatorname{gph} S) \text { with } z^{*} \in N_{+}(\bar{z} ; \operatorname{cl} \mathcal{L}(\bar{z})) \backslash\{0\}
$$

if $f$ is strictly Lipschitzian at $(\bar{x}, \bar{y})$. In the latter case, the SNC assumption on $f$ implies that the space $Z$ is finite-dimensional.

Proof. Given $(f, \prec, S)$ in the theorem, consider the set-valued mapping $S_{1}: Z \Rightarrow X \times Y \times Z$ and the set $S_{2} \subset X \times Y \times Z$ defined by

$$
S_{1}(z):=\operatorname{gph} S \times \mathcal{L}(z) \text { and } S_{2}:=\operatorname{gph} f .
$$

It is not hard to check that the point $(\bar{x}, \bar{y}, \bar{z})$ belongs to $S_{1}(\bar{z}) \cap S_{2}$ (by the local satiation property of the preference $\prec$ ) and happens to be locally extremal for the system $\left\{S_{1}, S_{2}\right\}$ at $(\bar{z}, 0)$ in the sense that there is a neighborhood $U$ of $(\bar{x}, \bar{y}, \bar{z})$ such that

$$
S_{1}(z) \cap S_{2} \cap U=\emptyset
$$

for any point $z \in \mathcal{L}(\bar{z})$ close to $\bar{z}$ but not equal to the latter by the preference $\prec$ nonreflexivity. This follows directly from the local optimality of $(\bar{x}, \bar{y})$ in (4.2) and the almost transitivity property of $\prec$; see [17, Example 5.65] for more details.

Since the spaces $X, Y$, and $Z$ are assumed to be Asplund, its product $X \times Y \times Z$ is Asplund as well, and we can apply to the system $\left\{S_{1}, S_{2}\right\}$ the extended extremal principle for multifunctions (see [20, Theorem 4.3] and [17, Theorem 5.68]). Note that the mapping $S_{1}$ is locally closed-graph (which is essential for the latter result), since the preference $\prec$ is assumed to be closed around $\bar{z}$. 
Given $\varepsilon>0$ and applying the afore-mentioned extremal principle to system (4.5) in $X \times Y \times Z$, find $z_{0} \in \bar{z}+\varepsilon \mathbb{B}_{Z} ;\left(x_{i}, y_{i}, z_{i}\right) \in(\bar{x}, \bar{y}, \bar{z})+\varepsilon \mathbb{B}_{X \times Y \times Z}$, and $\left(x_{i}^{*}, y_{i}^{*}, z_{i}^{*}\right) \in$ $X^{*} \times Y^{*} \times Z^{*}$ for $i=1,2$ such that $\left(x_{1}, y_{1}\right) \in \operatorname{gph} S, z_{1} \in \operatorname{cl} \mathcal{L}\left(z_{0}\right), z_{2}=f\left(x_{2}, y_{2}\right)$, and

$$
\begin{aligned}
& \left(x_{1}^{*}, y_{1}^{*}, z_{1}^{*}\right) \in \widehat{N}\left(\left(x_{1}, y_{1}, z_{1}\right) ; S_{1}\left(z_{0}\right)\right), \quad\left(x_{2}^{*}, y_{2}^{*}, z_{2}^{*}\right) \in \widehat{N}\left(\left(x_{2}, y_{2}, z_{2}\right) ; S_{2}\right), \\
& \left\|\left(x_{1}^{*}, y_{1}^{*}, z_{1}^{*}\right)+\left(x_{2}^{*}, y_{2}^{*}, z_{2}^{*}\right)\right\| \leq \varepsilon, \quad\left\|\left(x_{1}^{*}, y_{1}^{*}, z_{1}^{*}\right)\right\|+\left(x_{2}^{*}, y_{2}^{*}, z_{2}^{*}\right) \| \geq 1-\varepsilon
\end{aligned}
$$

Taking into account the structure of $\left\{S_{1}, S_{2}\right\}$ in (4.5) and using the product property $\widehat{N}\left(\cdot ; \Omega_{1} \times \Omega_{2}\right)=\widehat{N}\left(\cdot ; \Omega_{1}\right) \times \widehat{N}\left(\cdot ; \Omega_{2}\right)$ for Fréchet normals (which can be easily checked by definition), we get from (4.6) that

$$
\left(x_{1}^{*}, y_{1}^{*}\right) \in \hat{N}\left(\left(x_{1}, y_{1}\right) ; \operatorname{gph} S\right), \quad z_{1}^{*} \in \hat{N}\left(z_{1} ; \operatorname{cl} \mathcal{L}\left(z_{0}\right)\right), \quad\left(x_{2}^{*} ; y_{2}^{*}\right) \in \widehat{D}^{*} f\left(x_{2}, y_{2}\right)\left(-z_{2}^{*}\right)
$$

Now pick the sequence $\varepsilon:=1 / k$ as $k \rightarrow \infty$ and add the subindex " $k$ " to the corresponding elements above. By construction, we immediately have that $z_{0 k} \rightarrow \bar{z}$ and $\left(x_{i k}, y_{i k}, z_{i k}\right) \rightarrow(\bar{x}, \bar{y}, \bar{z})$ as $k \rightarrow \infty$ for $i=1,2$. Furthermore, by normalization if necessary, we can always suppose that the sequences $\left\{\left(x_{i k}^{*}, y_{i k}^{*}, z_{i k}^{*}\right)\right\} \subset X^{*} \times Y^{*} \times Z^{*}, i=1,2$, are bounded. Therefore, they are sequentially weak* compact in $X^{*} \times Y^{*} \times Z^{*}$ due to the Asplund property of $X \times Y \times Z$.; see [25]. Without loss of generality, suppose that

$$
\left(x_{i k}^{*}, y_{i k}^{*}, z_{i k}^{*}\right) \rightarrow\left(x_{i}^{*}, y_{i}^{*}, z_{i}^{*}\right) \in X^{*} \times Y^{*} \times Z^{*} \text { as } k \rightarrow \infty ; \quad i=1,2 .
$$

Passing to the limit in the first relationship of (4.7), we have

$$
\left(x_{2}^{*}, y_{2}^{*}, z_{2}^{*}\right)=-\left(x_{1}^{*}, y_{1}^{*}, z_{1}^{*}\right):=\left(x^{*}, y^{*},-z^{*}\right)
$$

Then we arrive to all the three inclusions in (4.3) by passing the limit in (4.8) and taking into account the definitions of the basic (2.1) and extended (4.1) normal cones.

Let us justify the nontriviality condition $\left(x^{*}, y^{*}, z^{*}\right) \neq 0$ under the SNC/ISNC assumptions made. To proceed by contradiction, suppose that $\left(x^{*}, y^{*}, z^{*}\right)=0$. Then

$$
\left(x_{1 k}^{*}, y_{1 k}^{*}, z_{1 k}^{*}\right) \stackrel{w^{*}}{\rightarrow} 0 \text { and }\left(x_{2 k}^{*}, y_{2 k}^{*}, z_{2 k}^{*}\right) \stackrel{w^{*}}{\rightarrow} 0 \text { as } k \rightarrow \infty \text {. }
$$

Assuming the SNC property of $f$ at $(\bar{x}, \bar{y})$, we have from (4.9) that $\left\|\left(x_{1 k}^{*}, y_{1 k}^{*}, z_{1 k}^{*}\right)\right\| \rightarrow 0$, which contradicts the second relationship in (4.7). On the other hand, if both the SNC assumption on $S$ at $(\bar{x}, \bar{y})$ and the ISNC assumption on $\mathcal{L}$ at $(\bar{z}, \bar{z})$ hold, then $\left\|\left(x_{2 k}^{*}, y_{2 k}^{*}, z_{2 k}^{*}\right)\right\| \rightarrow$ 0 , which contradicts $(4.7)$ as well.

To complete the proof of the theorem, it remains to consider the case when $f$ is strictly Lipschitzian at $(\bar{x}, \bar{y})$. In this case, the scalarization formula of $\{16$, Theorem 3.28] ensures that the first inclusion in (4.3) is equivalent to

$$
\left(x^{*}, y^{*}\right) \in \partial\left\langle z^{*}, f\right\rangle(\bar{x}, \bar{y})
$$

which implies that $\left(x^{*}, y^{*}\right)=0$ whenever $z^{*}=0$ and that (4.3) with $\left(x^{*}, y^{*}, z^{*}\right) \neq 0$ is equivalent to (4.4). Moreover, the SNC property of strictly Lipschitzian mappings implies 
the finite-dimensionality of the range space by [16, Corollary 3.30$]$.

Next consider EPECs defined in (4.2) with equilibrium constraints $y \in S(x)$ described by solution maps to the generalized equations (1.1). For simplicity, we present necessary conditions for optimal solutions to such problems when both mappings $f$ and $q$ are strictly Lipschitzian at $(\bar{x}, \bar{y})$.

Theorem 4.4 (necessary conditions for EPECs with generalized equation constraints and closed preferences). Let $(\bar{x}, \bar{y})$ be an optimal solution to the EPEC defined in (4.2) with the equilibrium constraints $y \in S(x)$ given by

$$
S(x):=\{y \in Y \mid 0 \in q(x, y)+Q(x, y)\}
$$

where $f: X \times Y \rightarrow Z$ and $q: X \times Y \rightarrow P$ are mapping between Asplund spaces that are strictly Lipschitzian at $(\bar{x}, \bar{y})$, where $\prec$ is a closed preference relation on $Z$, and where $Q: X \times Y \Rightarrow P$ is closed-graph around $(\bar{x}, \bar{y}, \bar{p})$ with $\bar{p}:=-q(\bar{x}, \bar{y}) \in Q(\bar{x}, \bar{y})$. Then there are $\left(z^{*}, p^{*}\right) \in Z^{*} \times P^{*}$, not both zero, satisfying

$$
0 \in \partial\left\langle z^{*}, f\right\rangle(\bar{x}, \bar{y})+\partial\left\langle p^{*}, q\right\rangle(\ddot{x}, \bar{y})+D_{N}^{*} Q(\bar{x}, \bar{y}, \bar{p})\left(p^{*}\right) \text { and } z^{*} \in N_{+}(\bar{z} ; \operatorname{cl} \mathcal{L}(\bar{z}))
$$

in each of the following two cases:

(a) $\operatorname{cl} \mathcal{L}$ is ISNC at $(\bar{z}, \bar{z})$, where $\bar{z}:=f(\bar{x}, \bar{y})$;

(b) $f$ is $S N C$ at $(\bar{x}, \bar{y})$ and either $Q$ is $S N C$ at $(\bar{x}, \bar{y}, \bar{p})$, or $\operatorname{dim} P<\infty$; in this case $Z$ must be finite-dimensional.

Proof. Based on Theorem 4.3, we need to obtain a upper estimate for the normal coderivative $D_{N}^{*} S(\bar{x}, \bar{y})$ of the solution map given in (3.12) and also to justify its SNC property at $(\bar{x}, \bar{y})$ in terms of $q$ and $Q$. The upper estimate (3.15) of $D_{N}^{*} S(\bar{x}, \bar{y})$ is established in the proof of Theorem 3.4 above provided the Fredholm qualification condition (3.16) and that either $Q$ is SNC at $(\bar{x}, \bar{y}, \bar{p})$, or $P$ is finite-dimensional. This allows us to justify, based on Theorem 4.3 and proceeding similarly to the proof of Theorem 3.4, the necessary optimality conditions given in (4.10) in case (b) of the theorem.

To prove the theorem in case (a), it is sufficient to show, in view of Theorem 4.3 and the above discussions, that the solution map $S$ in (3.12) is SNC at $(\bar{x}, \bar{y})$ provided that $Q$ is SNC at $(\bar{x}, \bar{y}, \bar{p}), g$ is locally Lipschitzian at $(\bar{x}, \bar{y})$, and the Fredholm qualification condition (3.16) is satisfied. To proceed, we observe that the graph of $S$ in (3.12) admits the inverse image representation

$$
\operatorname{gph} S=g^{-1}(\operatorname{gph} Q) \text { with } g(x, y):=(x, y,-q(x, y))
$$

Then apply [16, Theorem 3.84], which gives efficient conditions on the preservation of the SNC property under taking inverse images; this is a part of the SNC calculus strongly developed in [16]. It is not hard to check that the qualification condition of the latter theorem reduces, in the setting (4.11) under consideration, to the Fredholm qualification condition (3.16), while the SNC property of a set under the inverse image in [16, Theorem 3.84] is exactly the SNC property of $Q$ at $(\bar{x}, \bar{y}, \bar{p})$. Hence, to justify the SNC property of $S$ by $[16$, 
Theorem 3.84], we need to ensure that the mapping $g$ defined in (4.11) is PSNC at $(\bar{x}, \bar{y})$. The latter follows from the fact that any single-valued mapping locally Lipschitzian around some point is automatically PSNC at this point; see [16, Corollary 1.69]. The required Lipschitz continuity of $g$ in (4.11) is an obvious consequence of the assumed Lipschitzian property of the mapping $q$ around $(\bar{x}, \bar{y})$. This completes the proof of the theorem.

Having in hand Theorem 4.4, we can derive its specifications for the equilibrium constraints (1.1) given in each of the composite subdifferential forms (3.17). The formulations and proofs of results in this direction are similar to those presented in Section 3 for EPECs described via generalized order optimality on the upper level: they are fully based on the first-order and second-order calculus rules developed in [16].

\section{Optimality Conditions for MPECs via Exact Penalization}

The last section of this paper is devoted to the class of mathematical programs with equilibrium constraints (MPECs), which can be considered as a particular case of EPECs with respect to standard minimization of real-valued cost functions. On the other hand, specific features of minimizing real-valued functions make it possible to develop necessary optimality conditions for MPECs that do not have any counterparts in the case of EPECs and general problems of multiobjective/vector optimization.

In particular, specific results of the upper subdifferential type were developed by the author [14] for MPECs and other constraint minimization problems. Results of this type, which essentially exploit the nature of real-valued minimization, are significantly different from more conventional results of the lower subdifferential type in nonsmooth constrained minimization that are mainly based on the well-developed subdifferential calculus for basic subgradients (2.4); see [14, 17] for more details and discussions.

In this section, we follow another approach to (lower) subdifferential conditions for MPECs, which was developed by Ye and Ye [31] and Outrata [22] in finite-dimensional spaces. We consider here a general infinite-dimensional setting, which happens to be significantly more involved and leads us to a larger variety of results in comparison with MPECs in finite dimensions.

The underlying feature of this approach is a preliminary exact penalization of the constrained problems under consideration with subsequent applications of the subdifferential/coderivative calculus to penalized problems whose special structures are substantially different from the original ones and allow us to employ more suitable calculus results.

Let us start with formulating the calmness property of multifunctions [30], which essentially goes back to the "upper-Lipschitzian" property introduced by Robinson [26].

Definition 5.1 (calmness of set-valued mappings). Let $F: X \Rightarrow Y$ be a set-valued mapping between Banach spaces, and let $(\bar{x}, \bar{y}) \in \operatorname{gph} F$. Then $F$ is CALM at $(\bar{x}, \bar{y})$ with modulus $\ell \geq 0$ if there are neighborhoods $U$ of $\bar{x}$ and $V$ of $\bar{y}$ such that

$$
F(x) \cap V \subset F(\bar{x})+\ell\|x-\bar{x}\| \mathbb{B} \text { for all } x \in U \text {. }
$$


When $V=Y$ in (5.1); the calmness property of Definition 5.1 is known as Robinson's upper Lipschitzian property of $F$ at $\bar{x}$. The calmness terminology was suggested by Rockafellar and Wets [30], although the property itself was studied and applied earlier under different names; see, e.g., Ye and Ye [31].

One can see that the only difference between the calmness (5.1) and Aubin's Lipschitzlike (2.7) properties is that, instead of a pair of points $(x, u)$ independently varying in the neighborhood $U$ of the references point $\bar{x}$ in (2.7), only one point varies in (5.1) while the other is fixed being constantly equal to $\bar{x}$. In fact, calmness (5.1) is not a counterpart of the classical local Lipschitz continuity for the case of set-valued mappings; it does not reduce to the latter when $f$ is single-valued. This implies the non-robustness of the calmness/upper Lipschitzian properties (the sets $F(x)$ may even be empty near $\bar{x}$ ), in contrast to the Lipschitz-like/classical Lipschitzian ones. On the other hand, the requirement in (5.1) is less restrictive in comparison with that in (2.7). A major class of non-Lipschitzian while upper-Lipschitzian set-valued mappings between finite-dimensional spaces was discovered by Robinson [28] under the name of "piecewise polyhedral" multifunctions, i.e., those whose graph can be expressed as the union of finitely many convex polyhedral sets.

Note that the calmness/upper Lipschitzian notion for set-valued mappings in Definition 5.1 is closely related, for inverse mappings, to metric regularity at (not around) a point introduced by Ioffe and employed by him for exact penalization results in [10]; see also [17] for more details and applications of this property under the name of "weakened metric regularity." In fact, the following lemma proved by Ye and Ye [31] for calm mappings and earlier by Zhang [33] for upper Lipschitzian ones in a more special case, is largely similar to the penalization/reduction theorem by Ioffe [10] established in somewhat different setting. The reader can find more results and information about applications of calmness and related properties in the recent paper by Henrion and Outrata [8] and the references therein.

Lemma 5.2 (exact penalization under generalized equation constraints). Let $\bar{t}$ be a local optimal solution to the problem:

$$
\text { minimize } \varphi(t) \text { subject to } 0 \in F(t), t \in \Omega
$$

where $\varphi: T \rightarrow \mathbb{R}$ and $F: T \Rightarrow Z$ in the framework of Banach spaces. Assume that $\varphi$ is locally Lipschitzian around $\bar{t}$ with modulus $\ell_{\varphi}$ and that the mapping

$$
\left(F^{-1} \cap \Omega\right)(z):=F^{-1}(z) \cap \Omega
$$

is calm at $(0, \bar{t})$ with modulus $\ell$. Then there are neighborhoods $V$ of $\bar{t}$ and $U$ of $0 \in Z$ such that $(\bar{t}, 0) \in T \times Z$ solves the penalized problem:

$$
\text { minimize } \psi(t, z):=\varphi(t)+\mu\|z\| \text { subject to } z \in F(t) \cap U, t \in \Omega \cap V
$$

provided that $\mu \geq \ell_{\varphi} \cdot \ell$.

Observe that the major constraint $0 \in F(t)$ in (5.2) is given in the form of nonparametric generalized equations; and thus problem (5.2) can be viewed as an abstract MPEC. The next result; providing necessary optimality conditions for the abstract MPECs (5.2), is based on applying the generalized differential and SNC calculi [16] to the penalized problem (5.4). 
Theorem 5.3 (necessary optimality conditions for abstract MPECs). Let $\bar{t}$ be a local optimal solution to the abstract MPEC given in (5.2), where the spaces $T$ and $Z$ are Asplund and where the sets $\Omega$ and $\operatorname{gph} F$ are locally closed around $\bar{t}$ and $(\bar{t}, 0)$, respectively. Assume that $\varphi$ is locally Lipschitzian around $\bar{t}$ with modulus $\ell_{\varphi}$, that $F^{-1} \cap \Omega$ defined in (5.3) is calm at $(0, \tilde{t})$ with modulus $\ell$, and that the mixed qualification condition

$$
D_{M}^{*} F(\bar{t}, 0)(0) \cap(-N(\bar{t} ; \Omega))=\{0\}
$$

is satisfied. Suppose also that either $F$ is PSNC at $(\bar{t}, 0)$, or $\Omega$ is SNC at $\bar{t}$. Then for any $\mu \geq \ell_{\varphi} \cdot \ell$ there is $z^{*} \in Z^{*}$ with $\left\|z^{*}\right\| \leq \mu$ such that

$$
0 \in \partial \varphi(\bar{t})+D_{N}^{*} F(\bar{t}, 0)\left(z^{*}\right)+N(\bar{t} ; \Omega)
$$

Proof. By the exact penalization result of Lemma 5.2, the point $(\bar{t}, 0) \in T \times Z$ provides a local optimal solution to the penalization problem (5.4) with $\mu \geq \ell_{\varphi} \cdot \ell$. Since both sets $U$ and $V$ are open in problem (5.4), it can be equivalently (from the viewpoint of necessary optimality conditions) written as:

$$
\text { minimize } \varphi(t)+\mu\|z\| \text { subject to } z \in F(t), \quad t \in \Omega \text {. }
$$

In turn, the latter problem is equivalent to the minimization problem with geometric constraints given as the intersection of two sets:

$$
\text { minimize } \varphi(t)+\mu\|z\| \text { subject to }(t, z) \in \operatorname{gph} F \cap(\Omega \times Z) \text {, }
$$

which can be written as the following unconstrained problem with an infinite penalty via the indicator function of a set:

$$
\operatorname{minimize} \varphi(t)+\mu\|z\|+\delta((t, z) ; \operatorname{gph} F \cap(\Omega \times Z))
$$

where $\delta(x ; \Lambda):=0$ if $x \in \Lambda$ and $\delta(x ; \Lambda):=\infty$ otherwise. Applying the generalized Fermat rule of $[16$, Proposition 1.114] to the optimal solution $(\bar{t}, 0)$ of $(5.7)$, we have

$$
0 \in \partial(\varphi(\cdot)+\mu\|\cdot\|+\partial \delta(\cdot ; \operatorname{gph} F \cap(\Omega \times Z)))(\bar{t}, 0)
$$

Now using (twice) the subdifferential sum rule of [16, Theorem $2.33(\mathrm{c})]$ in (5.8) with taking into account that both $\varphi$ and $\|\cdot\|$ are locally Lipschitzian around $(\bar{t}, 0)$ and that

$$
\partial \delta(\bar{x} ; \Lambda)=N(\bar{x} ; \Lambda) \text { as } \bar{x} \in \Lambda, \quad \partial\|\cdot\|(z) \subset \mathbb{B}^{*} \text { for any } z \in Z,
$$

we arrive at the inclusion

$$
(0,0) \in(\partial \varphi(\bar{t}), 0)+\mu\left(0, \mathbb{B}^{*}\right)+N((\bar{t}, 0) ; \operatorname{gph} F \cap(\Omega \times Z)) .
$$

To proceed, we need representing the normal cone to the set intersection in (5.9) via generalized differential constructions to the initial data $F$ and $\Omega$ of the original problem (5.2) under appropriate qualification and SNC conditions. Observe that both sets from the intersection in (5.9) belong to the product of Asplund spaces $T \times Z$. Applying the basic intersection 
rule in product spaces from [16, Theorem 3.4] and taking into account the structure of the second set $\Omega \times Z$ in the intersection, we get

$$
N((\bar{t}, 0) ; \operatorname{gph} F \cap(\Omega \times Z)) \subset N((\bar{t}, 0) ; \operatorname{gph} F)+N(\bar{t} ; \Omega) \times\{0\}
$$

providing the "limiting qualification condition" of the latter theorem and that either $F$ is PSNC at $(\bar{t}, 0)$, or $\Omega$ is SNC at $\bar{t}$. It is not hard to check that the afore-mentioned limiting qualification condition of [16, Theorem 3.4] is ensured, due to the structures of the sets gph $F$ and $\Omega \times Z$ in the intersection (5.9), by the qualification condition (5.5) of this theorem involving the mixed coderivative $D_{M}^{*} F(\bar{t}, 0)$. It remains to observe that (5.9) and (5.10) imply, due to definition (2.2) of the normal coderivative, the necessary optimality condition (5.6) with $\left\|z^{*}\right\| \leq \mu$. This completes the proof of the theorem.

Note that $F$ is automatically PSNC at $(\bar{t}, 0)$ and the qualification condition (5.5) holds by $(2.8)$ if $F$ is Lipschitz-like around $(\bar{t}, 0)$. This is due to the characterization of the Lipschitz-like property from [16, Theorem 4.10]. On the other hand. the calmness assumption of Theorem 5.3 holds also for problems with no Lipschitz-like assumption of $F$; see, e.g., the results in Outrata [22] based on fundamental Robinson's developments [28] for calmness/upper Lipschitzian properties of piecewise polyhedral multifunctions.

The following consequence of Theorem 5.3 and further rules of generalized differential and SNC calculi addresses abstract MPECs with the constraint mapping $F$ of a special structure important for the subsequent application to the equilibrium constraints governed by parametric generalized equations/variational conditions.

Corollary 5.4 (necessary conditions for abstract MPECs of special structure). Suppose that the constraint mapping in (5.2) admits the representation

$$
F(t)=g(t)+\theta
$$

in the framework of Theorem 5.3, where $g: T \rightarrow Z$ is continuous around $\bar{t}$ and $\theta \subset Z$ is locally closed around $\bar{z}:=-g(\bar{t})$ in addition to the general requirements on $\varphi, \Omega$ and the calmness property of $F^{-1} \cap \Omega$ in the theorem. Impose the qualification condition

$$
D_{M}^{*} g(\bar{t})(0) \cap(-N(\bar{t} ; \Omega))=\{0\}
$$

and assume that either the set $\Omega$ is SNC at $\bar{x}$, or the mapping $g$ is PSNC at this point; the latter property together with (5.12) are automatic when $g$ is locally Lipschitzian around $\bar{t}$. Then there is $z^{*} \in-N(\bar{x} ; \theta)$ with $\left\|z^{*}\right\| \leq \mu$ as $\mu \geq \ell_{\varphi} \cdot \ell$ such that

$$
0 \in \partial \varphi(\bar{t})+D_{N}^{*} g(\bar{t})\left(z^{*}\right)+N(\bar{t} ; \Omega),
$$

which is equivalent to

$$
0 \in \partial \varphi(\bar{t})+\partial\left\langle z^{*}, g\right\rangle(\bar{t})+N(\bar{t} ; \Omega)
$$

if the mapping $g$ is strictly Lipschitzian at. $\bar{t}$. 
Proof. Considering the constant mapping $\Theta(t) \equiv \Theta$ for all $t \in T$, it easy to see that it is PSNC at any point $(\bar{t}, \bar{\theta}) \in T \times \Theta$ and that both coderivatives $D^{*}=D_{N}^{*}, D_{M}^{*}$ of this mappings are represented in the form

$$
D^{*} \Theta(\bar{t}, \bar{\theta})\left(z^{*}\right)= \begin{cases}0 & \text { if }-z^{*} \in N(\bar{\theta} ; \Theta) \\ \emptyset & \text { otherwise }\end{cases}
$$

Then applying the coderivative sum rule from [16, Theorem 3.10] to both coderivatives $D^{*}=D_{N}^{*}, D_{M}^{*}$ of the sum $g+\Theta$ in (5.11), we get

$$
D^{*} F(\bar{t}, 0)\left(z^{*}\right) \subset \begin{cases}D^{*} g(\bar{t})\left(z^{*}\right) & \text { if }-z^{*} \in \dot{N}(\bar{z} ; \theta) \\ \emptyset & \text { otherwise. }\end{cases}
$$

Substituting (5.14) into (5.5) and (5.6), we arrive at (5.12) and (5.13), respectively. To complete the proof of the corollary, it remains to observe that the PSNC property of $g$ at $\bar{t}$ implied the one for $F$ in $(5.11)$ at $(\bar{t}, 0)$ by the preservation/calculus result for the PSNC property of sums established in [16, Theorem 3.88].

Next let us consider the MPEC given in the form:

$$
\text { minimize } \varphi(x, y) \text { subject to } 0 \in q(x, y)+Q(x, y), \quad(x, y) \in \Omega
$$

with equilibrium constraints governed by parametric generalized equations and also with geometric constraints depending on both variables $(x, y)$; note that the latter constraints are additional in comparison with the previous EPEC studies in Sections 3 and 4.

Theorem 5.5 (necessary optimality conditions for MPECs with generalized equation and geometric constraints). Let $(\bar{x}, \bar{y})$ be a local optimal solution to the MPEC given in (5.15), where $q: X \times Y \rightarrow Z$ and $Q: X \times Y \rightrightarrows Z$ are mappings between Asplund spaces. Assume that $\varphi: X \times Y \rightarrow \mathbb{R}$ is Lipschitz continuous around $(\bar{x}, \bar{y})$ with modulus $\ell_{\varphi}$, that $q$ is continuous around this point, and that the sets $\Omega$ and $\operatorname{gph} Q$ are locally closed around $(\bar{x}, \bar{y})$ and $(\bar{x}, \bar{y}, \bar{z})$ with $\bar{z}:=-q(\bar{x}, \bar{y})$, respectively. Suppose also that the mapping $G: X \times Y \times Z \Rightarrow X \times Y$ given by

$$
G(u, v, w):=\{(x, y) \in \Omega \mid(u+x, v+y, w-q(x, y)) \in \operatorname{gph} Q\}
$$

is calm at $(0,0,0, \bar{x}, \bar{y})$ with modulus $\ell$, that the qualification condition

$$
D_{M}^{*} q(\bar{x}, \bar{y})(0) \cap(-N((\bar{x}, \bar{y}) ; \Omega))=\{0\}
$$

is fulfilled, and that either $q$ is PSNC at $(\bar{x}, \bar{y})$ or $\Omega$ is SNC at this point.

Then there are dual elements $\left(x^{*}, y^{*}, z^{*}\right) \in X^{*} \times Y^{*} \times Z^{*}$ with $\left\|\left(x^{*}, y^{*}, z^{*}\right)\right\| \leq \ell_{\varphi} \cdot \ell$ and $\left(x^{*}, y^{*}\right) \in D_{N}^{*} Q(\bar{x}, \bar{y}, \bar{z})\left(z^{*}\right)$ satisfying

$$
\left(-x^{*},-y^{*}\right) \in \partial \varphi(\bar{x}, \bar{y})+D_{N}^{*} q(\bar{x}, \bar{y})\left(z^{*}\right)+N((\bar{x}, \bar{y}) ; \Omega),
$$

which implies that

$$
0 \in \partial \varphi(\bar{x}, \bar{y})+D_{N}^{*} q(\bar{x}, \bar{y})\left(z^{*}\right)+D_{N}^{*} Q(\bar{x}, \bar{y}, \bar{z})\left(z^{*}\right)+N((\bar{x}, \bar{y}) ; \Omega)
$$


Proof. We reduce problem (5.15) to the one considered in Corollary 5.4 with the data $t=(x, y) \in X \times Y$ and

$$
F(x, y):=g(x, y)+\Theta, \quad \Theta:=\operatorname{gph} Q, g(x, y):=(-x,-y, q(x, y))
$$

Applying Corollary 5.4 to the above problem with data (5.19) and taking into account that

$$
g(x, y)=(-x,-y, 0)+(0,0, q(x, y))
$$

we conclude from [16, Theorem 1.70] that $g$ is PSNC at $(\bar{x}, \bar{y})$ if and only if $f$ is PSNC at this point. Then the coderivative sum rule from [16, Theorem 1.62(ii)] gives

$$
D^{*} g(\bar{x}, \bar{y})\left(x^{*}, y^{*}, z^{*}\right)=\left(-x^{*},-y^{*}\right)+D^{*} q(\bar{x}, \bar{y})\left(z^{*}\right)
$$

for both coderivatives $D^{*}=D_{N}^{*}, D_{M}^{*}$. Substituting (5.20) into the relationships of Corollary 5.4 and taking into account the special structure of $(5.19)$, we arrive all the qualification and necessary optimality conditions of the theorem. Observe finally that the mapping (5.3) in Corollary 5.4 reduces to $G$ in $(5.16)$ for the data (5.19) under consideration.

As usual, the strict Lipschitzian assumption on the base mapping $q$ in the generalized equation (5.15) allows us to specify and simplify the results obtained in Theorem 5.5.

Corollary 5.6 (optimality conditions for MPECs with strictly Lipschitzian bases in generalized equation constraints). Suppose in the general framework of Theorem 5.5 that $q$ is strictly Lipschitzian at $(\bar{x}, \bar{y})$, that $Q$ is SNC at $(\bar{x}, \bar{y}, \bar{z})$, and that the relation

$$
\left(x^{*}, y^{*}\right) \in\left[\partial\left\langle z^{*}, q\right\rangle(\bar{x}, \bar{y})+N((\bar{x}, \bar{y}) ; \Omega)\right] \cap\left(-D_{N}^{*} Q(\bar{x}, \bar{y}, \bar{z})\left(z^{*}\right)\right) .
$$

holds only for $x^{*}=y^{*}=z^{*}=0$. Then there is $z^{*} \in Z^{*}$ such that one has the condition

$$
0 \in \partial \varphi(\bar{x}, \bar{y})+\partial\left\langle z^{*}, q\right\rangle(\bar{x}, \bar{y})\left(z^{*}\right)+D_{N}^{*} Q(\bar{x}, \bar{y}, \bar{z})\left(z^{*}\right)+N((\bar{x}, \bar{y}) ; \Omega) .
$$

Proof. Considering the mapping $h: X \times Y \times Z \times X \times Y \rightarrow X \times Y \times Z$ defined by

$$
h(u, v, w, x, y):=(u+x, v+y, w-q(x, y)),
$$

we represent $G$ from (5.16) in the form of the constraint system:

$G(u, v, w)=\{(x, y) \in X \times Y \mid h(u, v, w, x, y) \in \operatorname{gph} Q, \quad(u, v, w, x, y) \in X \times Y \times Z \times \Omega\}$.

It is not hard to check, due to the special structure of $h$ and $G$, that the assumptions made in the corollary ensure by [16, Corollary 4.41] that the mapping $G$ is Lipschitz-like around $(0,0,0, \bar{x}, \bar{y})$, and hence it is calm at this point. Since the qualification condition (5.17) is automatic for Lipschitzian mappings, the optimality condition (5.21) follows from (5.18) by the scalarization formula of [16, Theorem 3.28].

Similarly to the results of Section 3, we can derive specifications of necessary optimality and qualification conditions of Theorem 5.5 and Corollary 5.6 for the cases of MPECs with equilibrium constraints governed by parametric generalized equations with composite subdifferential structures given in each of the forms (5.17). 


\section{References}

[1] Aubin, J.-P.: Lipschitz behavior of solutions to convex minimization problems. Math. Oper. Res. 9, 87-111 (1984)

[2] Borwein, J.M., Strójwas, H.M.: Tangential approximations. Nonlinear Anal. 9, 1347-1366 (1985)

[3] Dempe, S.: Annotated bibliography on bilevel programming and mathematical programs with equilibrium constraints. Optimization 52, 333-359 (2003)

[4] Dontchev, A.L., Rockafellar, R.T.: Characterizations of strong regularity for variational inequalities over polyhedral convex sets. SIAM J. Optim. 7, 1087-1105 (1996)

[5] Fabian, M., Mordukhovich, B.S.: Sequential normal compactness versus topological normal compactness in variational analysis. Nonlinear Anal. 54, 1057-1067 (2003)

[6] Facchinei, F., Pang, J.-S.: Finite-Dimensional Variational Inequalities and Complementary Problems. Springer Series in Operations Research, Springer, New York, 2003

[7] Fukushima, M., Pang, J.-S.: Quasi-variational inequalities, generalized Nash equilibria, and multi-leader-follower games. Comput. Management Sci. 1, 21-56 (2005)

[8] Henrion, R., Outrata, J.V.: Calmness of constraint systems with applications. Math. Prog. 104, 437-464 (2006)

[9] Hu X., Ralph, D.: Using EPECs to model bilevel games in restructured electricity markets with locational prices. The Judge Institute of Management, University of Cambridge, UK. Preprint (2004)

[10] Ioffe, A.D.: Necessary and sufficient conditions for a local minimum, I: A reduction theorem and first order conditions. SIAM J. Control Optim. 17, 245-250 (1979)

[11] Jahn, J.: Vector Optimization: Theory, Applications and Extensions. Springer Series in Operations Research, Springer, Berlin, 2004

[12] Jourani, A., Thibault, L.: Verifiable conditions for openness, metric regularity of multivalued mappings. Trans. Amer. Math. Soc. 347, 1225-1268 (1995)

[13] Luo, Z.Q., Pang, J.-S., Ralph, D.: Mathematical Programs with Equilibrium Constraints. Cambridge University Press, Cambridge, UK, 1996

[14] Mordukhovich, B.S.: Necessary conditions in nonsmooth minimization via lower and upper subgradients. Set-Valued Anal. 12, 163-193 (2004)

[15] Mordukhovich, B.S.: Equilibrium problems with equilibrium constraints via multiobjective optimization. Optim. Meth. Soft. 19, 479-492 (2004)

[16] Mordukhovich, B.S.: Variational Analysis and Generalized Differentiation, I: Basic Theory. Grundlehren Series (Fundamental Principles of Mathematical Sciences) 330, Springer, Berlin, 2006

[17] Mordukhovich; B.S.: Variational Analysis and Generalized Differentiation, II: Applications. Grundlehren Series (Fundamental Principles of Mathematical Sciences) 331, Springer, Berlin, 2006

[18] Mordukhovich, B.S., Outrata, J.V.: Second-order subdifferentials and their applications. SIAM J. Optim. 12, 139-169 (2001) 
[19] Mordukhovich, B.S., Outrata, J.V., Červinka, M.: Equilibrium problems with complementarity constraints: case study with applications to oligopolistic markets. Optimization, to appear.

[20] Mordukhovich, B.S., Treiman, J.S., Zhu, Q.J.: An extended extremal principle with applications to multiobjective optimization. SIAM J. Optim. 14, 359-379 (2003)

[21] Mordukhovich, B.S., Wang, B.: Generalized differentiation for moving objects. In: 'Optimal Control, Stabilization and Nonsmooth Analysis,' de Queiroz/Malisoff/Wolenski (eds.)., Lecture Notes Cont. Inf. Sci. 301, 351-361, Springer, New York, 2004

[22] Outrata, J.V.: A generalized mathematical program with equilibrium constraints. SLAM J: Control Optim. 38, 1623-1638 (2000)

[23] Outrata, J.V.: A note on a class of equilibrium problems with equilibrium constraints. Kybernetika 40, 585-594 (2004)

[24] Outrata, J.V., Kočvara, M., Zowe, J.: Nonsmooth Approach to Optimization Problems with Equilibrium Constraints. Kluwer, Dordrecht, The Netherlands, 1998

[25] Phelps, R.R.: Convex Functions, Monotone Operators and Differentiability, 2nd edition. Lecture Notes in Mathematics 1364, Springer, Berlin, 1993

[26] Robinson, S.M.: Generalized equations and their solutions, I: basic theory. Math. Prog. Study 10, 128-141 (1979)

[27] Robinson, S.M.: Strongly regular generalized equations. Math. Oper. Res. 5, 43-62 (1980)

[28] Robinson, S.M.: Some continuity properties of polyhedral multifunctions. Math. Prog. Study 14, 206-214 (1981)

[29] Robinson, S.M.: Variational conditions with smooth constraints: structure and analysis. Math. Prog. 97, 245-265 (2003)

[30] Rockafellar, R.T., Wets, R.J.-B.: Variational Analysis. Grundlehren Series (Fundamental Principles of Mathematical Sciences) 317, Springer, Berlin, 1998

[31] Ye, J.J., Ye, X.Y.: Necessary optimality conditions for optimization problems with variational inequality constraints. Math. Oper. Res. 22, 977-997 (1997)

[32] Ye, J.J., Zhu, Q.J.: Multiobjective optimization problems with variational inequality constraints. Math. Prog. 96, 139-160 (2003)

[33] Zhang, R.: Problems of hierarchical optimization in finite dimensions. SIAM J. Optim. 4, 521-536 (1994) 